TRANSACTIONS OF THE

AMERICAN MATHEMATICAL SOCIETY

Volume 358, Number 12, December 2006, Pages 5441-5483

S 0002-9947(06)03871-2

Article electronically published on July 21, 2006

\title{
DEFORMATION THEORY OF ABELIAN CATEGORIES
}

\author{
WENDY LOWEN AND MICHEL VAN DEN BERGH
}

\begin{abstract}
In this paper we develop the basic infinitesimal deformation theory of abelian categories. This theory yields a natural generalization of the wellknown deformation theory of algebras developed by Gerstenhaber. As part of our deformation theory we define a notion of flatness for abelian categories. We show that various basic properties are preserved under flat deformations, and we construct several equivalences between deformation problems.
\end{abstract}

\section{INTRODUCTION}

In this paper we develop the basic infinitesimal deformation theory of abelian categories. This theory yields a natural generalization of the well-known deformation theory of algebras developed by Gerstenhaber [7, 9, 10. In a subsequent paper [17. we will develop the corresponding obstruction theory in terms of a suitable notion of Hochschild cohomology.

Deformation theory of abelian categories is important for non-commutative algebraic geometry. One of the possible goals of non-commutative algebraic geometry is to understand the abelian (or triangulated) categories which have properties close to those of the (derived) category of (quasi-)coherent sheaves on a scheme. One is particularly interested in those properties which are preserved under suitable deformations. The deformation theory of (abstract) triangulated categories seems at this point somewhat elusive (due to the unclear status of the currently accepted axioms), but, as we will show in this paper, there is a perfectly good deformation theory for abelian categories.

As in any deformation theory we need some kind of flatness in order to control the deformed objects. Therefore the first contribution of this paper is a notion of flatness for abelian categories (see \$3). To the best of our knowledge this definition is new.

In the rest of this Introduction $R$ is a commutative coherent ring. We will consider $R$-linear abelian categories. Informally these may be viewed as noncommutative schemes over the (commutative) affine base scheme Spec $R$.

Let $\mathcal{C}$ be an $R$-linear abelian category. Our notion of flatness has the following properties:

(1) if $A$ is an $R$-algebra, then $\operatorname{Mod}(A)$ is flat if and only if $A$ is flat over $R$;

(2) $\mathcal{C}$ is flat if and only if $\mathcal{C}^{\text {op }}$ is flat;

Received by the editors September 24, 2004 and, in revised form, October 7, 2004.

2000 Mathematics Subject Classification. Primary 13D10, 14A22, 18E15.

The first author is a Postdoctoral Fellow of the Research Foundation-Flanders (FWO).

The second author is a senior researcher at the FWO. 
(3) if $\mathcal{C}$ has enough injectives, $\mathcal{C}$ is flat if and only if injectives are flat [3] in $\mathcal{C}^{\mathrm{op}}$

(4) if $\mathcal{C}$ is essentially small, $\mathcal{C}$ is flat if and only if $\operatorname{lnd}(\mathcal{C})$ is flat (recall that $\operatorname{Ind}(\mathcal{C})$ is the formal closure of $\mathcal{C}$ under filtered colimits (see 92.2 ), it is a category with enough injectives);

(5) flatness is stable under "base change" (see below).

By enlarging the universe (see \$2.1) we may assume that any category is small. Therefore, in principle, we could take properties (3) and (4) as the definition of flatness. However this would make the self duality (property (2)) very obscure. Our definition of flatness is somewhat more technical (see \$3), but it is manifestly left-right symmetric.

Of fundamental importance in algebraic geometry is the concept of base change. There is a natural substitute for this notion in our setting. Consider a morphism $\theta: R \longrightarrow S$ between coherent rings such that $S$ is finitely presented over $R$, and let $\mathcal{C}$ be an $R$-linear category. The category $\mathcal{C}_{S}$ is the category of $S$-objects in $\mathcal{C}$, i.e. the pairs $(C, \varphi)$ where $C \in \mathrm{Ob}(\mathcal{C})$ and $\varphi: S \longrightarrow \mathcal{C}(C, C)$ is an $R$-algebra map. Intuitively $\mathcal{C}_{S}$ the base extension of the "non-commutative scheme" $\mathcal{C}$ to Spec $S$. We show in 4 that base change is compatible with various natural constructions such as Ind and Mod.

Assume now that $\theta$ is surjective such that $I \stackrel{\text { def }}{=} \operatorname{ker} \theta$ satisfies $I^{n}=0$ for some $n$. The surjectivity of $\theta$ implies that $\mathcal{C}_{S}$ is a full abelian subcategory of $\mathcal{C}$.

Let $\mathcal{D}$ be a flat $S$-linear category. A flat $R$-deformation of $\mathcal{D}$ is, roughly speaking, a flat lift of $\mathcal{D}$ along the functor $(-)_{S}$. In $\$ 6$ we show that some of the basic properties of abelian categories are preserved under flat deformation. More precisely, we show that the following properties of $\mathcal{D}$ lift to a flat deformation:

(1) $\mathcal{D}$ is essentially small;

(2) $\mathcal{D}$ has enough injectives;

(3) $\mathcal{D}$ is a Grothendieck category (i.e. a cocomplete abelian category with a generator and exact filtered colimits);

(4) $\mathcal{D}$ is a locally coherent Grothendieck category (i.e. $\mathcal{D}$ is Grothendieck and is generated by a small abelian subcategory of finitely presented objects).

In addition we show (see Theorem 8.5) that up to equivalence the number of flat deformations of an essentially small, respectively a Grothendieck, category is small.

Flatness is necessary for some of these properties. For example if $k$ is a field, then there are non-flat deformations of $\operatorname{Mod}(k)$ which do not have enough injectives, (Example 6.17) and furthermore the number of non-flat deformations is not small (Remark 8.6).

In 97 we discuss the compatibility of localization with deformations. Among other things we show that a deformation of $\mathcal{D}$ gives rise to deformations of all its localizations (Theorem 7.1).

As a preparation to the sequel of this paper in which we will develop the obstruction theory of abelian categories, we study the associated deformation functors in 88. By $\operatorname{Def}_{\mathcal{D}}(R)$ we denote the flat $R$-deformations of $\mathcal{D}$.

We have the following results:

(1) If $\mathcal{D}$ is an essentially small flat $S$-linear category, then there is an equivalence between $\operatorname{Def}_{\mathcal{D}}(R)$ and $\operatorname{Def}_{\text {Ind }(\mathcal{D})}(R)$. 
(2) If $\mathcal{D}$ is a locally coherent flat $S$-linear Grothendieck category, then there is an equivalence between $\operatorname{Def}_{\mathcal{D}}(R)$ and $\operatorname{Def}_{\mathrm{Fp}(\mathcal{D})}(R)$, where $\operatorname{Fp}(\mathcal{D})$ is the full (abelian) subcategory of $\mathcal{D}$ consisting of the finitely presented objects.

In order to describe some more results of 88 we also need to introduce deformations of general $R$-linear categories. This is done by considering such categories as "rings with several objects" [19]. We denote by $\operatorname{def}_{\mathfrak{b}}(R)$ the flat $R$-deformations of $\mathfrak{b}$ as an $R$-linear category. Note that deforming an $R$-linear abelian category $\mathcal{D}$ as an abelian category is completely different from deforming $\mathcal{D}$ as an $R$-linear category.

We prove the following results:

(3) If $\mathfrak{b}$ is an essentially small flat $S$-linear category, then then there is an equivalence between $\operatorname{def}_{\mathfrak{b}}(R)$ and $\operatorname{Def}_{\operatorname{Mod}(\mathfrak{b})}(R)$, where $\operatorname{Mod}(\mathfrak{b})$ is the category of covariant additive functors from $\mathfrak{b}$ to $A b$. In particular if $B$ is a flat $S$-algebra, then there is an equivalence between the deformations of $B$ and the deformations of $\operatorname{Mod}(B)$.

(4) If $\mathcal{D}$ is a flat $S$-linear category with enough injectives, then there is an equivalence between $\operatorname{Def}_{\mathcal{D}}(R)$ and $\operatorname{def}_{\operatorname{lnj}(\mathcal{D})}(R)$, where $\operatorname{Inj}(\mathcal{D})$ denotes the full (additive) subcategory of $\mathcal{D}$ of injective objects.

Property (3) shows that indeed our deformation theory generalizes the deformation theory of algebras.

In the final section of this paper we apply our methods to the deformations of the category $\operatorname{Mod}\left(\mathcal{O}_{X}\right)$ of sheaves of modules over a ringed space $\left(X, \mathcal{O}_{X}\right)$. For simplicity of exposition we assume here that $\mathcal{O}_{X}$ is a sheaf of $k$-algebras where $k$ is a field. Assume that $X$ has a basis $\mathcal{B}$ satisfying the following acyclicity condition:

$$
\forall U \in \mathcal{B}: H^{i}\left(U, \mathcal{O}_{U}\right)=0 \quad \text { for } i=1,2 .
$$

Let $\mathcal{O}_{\mathcal{B}}$ be the restriction of $\mathcal{O}_{X}$ to $\mathcal{B}$ and let $\operatorname{PreMod}\left(\mathcal{O}_{\mathcal{B}}\right)$ be the corresponding category of presheaves. We show that there is an equivalence

$$
\operatorname{Def}_{\operatorname{PreMod}\left(\mathcal{O}_{\mathcal{B}}\right)}(R) \cong \operatorname{Def}_{\operatorname{Mod}\left(\mathcal{O}_{X}\right)}(R) \text {. }
$$

Let $\mathfrak{u}$ be the pre-additive category spanned by the (presheaf) extensions by zero of the $\mathcal{O}_{U}$ for $U \in \mathcal{B}$. In other words we may take $\operatorname{Ob}(\mathfrak{u})=\mathcal{B}$, and we have

$$
\mathfrak{u}(U, V)=\left\{\begin{aligned}
\mathcal{O}_{X}(U) & \text { if } U \subset V, \\
0 & \text { otherwise. }
\end{aligned}\right.
$$

Using property (3) above, it is easy to see that

$$
\operatorname{Def}_{\operatorname{PreMod}\left(\mathcal{O}_{\mathcal{B}}\right)}(R) \cong \operatorname{def}_{\mathfrak{u}}(R) .
$$

These results confirm the fundamental insight of Gerstenhaber and Schack 6, 8. that one should define the deformations of a ringed space $\left(X, \mathcal{O}_{X}\right)$ not as the deformations of $\mathcal{O}_{X}$ as a sheaf of $k$-algebras, but rather as the deformations of the $k$-linear category $\mathfrak{u}$ (or of the "diagram" $\left(\mathcal{B}, \mathcal{O}_{\mathcal{B}}\right)$ in case $\left.X \in \mathcal{B}\right)$. These "virtual" deformations are nothing but the deformations of the abelian category $\operatorname{Mod}\left(\mathcal{O}_{X}\right)$.

\section{Preliminaries}

2.1. Universes. It is well known [18] that category theory needs some extension of the Zermelo-Fraenkel axioms of set theory (ZF). One possible extension is given by the Gödel-Bernays axioms (GB) which incorporate classes into set theory. This 
makes it possible to introduce the category Set while at the same time avoiding Russel's paradox.

This solution is not entirely satisfying since for example one would also like to talk about Cls, the category of all classes, and there is no room for this notion in GB. In particular, in the deformation theory of categories we consider below, this seems to lead to foundational problems.

To solve such problems Grothendieck introduced a more flexible extension of the Zermelo-Fraenkel system: the theory of universes [2. The theory of universes does not introduce new types of objects but adds the universe axiom (U) below.

A universe $\mathcal{U}$ is a set with the following properties:

(1) if $x \in \mathcal{U}$ and if $y \in x$, then $y \in \mathcal{U}$;

(2) if $x, y \in \mathcal{U}$, then $\{x, y\} \in \mathcal{U}$;

(3) if $x \in \mathcal{U}$, then the powerset $\mathcal{P}(x)$ of $x$ is in $\mathcal{U}$;

(4) if $\left(x_{i}\right)_{i \in I}$ is a family of objects in $\mathcal{U}$ indexed by an element of $\mathcal{U}$, then $\bigcup_{i \in I} x_{i} \in \mathcal{U}$

(5) if $U \in U$ and $f: U \longrightarrow \mathcal{U}$ is a function, then $\{f(x) \mid x \in U\} \in \mathcal{U}$.

Note that $(x, y)$ is defined as $\{\{x, y\}, x\}$, and hence if $x, y \in \mathcal{U}$, then so is $(x, y)$. A universe $\mathcal{U}$ with $\mathbb{N} \in \mathcal{U}$ is itself a model for ZF.

As the only known non-empty universe contains only finite sets, we need the following new axiom:

(U) every set is the element of a universe.

In this paper, we will work with ZFCU (the ZF axioms + the axiom of choice + the universe axiom). By requiring $\{x, \mathbb{N}\} \in \mathcal{U}$, every set $x$ is the element of a universe containing $x$ and $\mathbb{N}$. From now on, by a universe we will always mean a universe containing $\mathbb{N}$. In particular, every such universe is itself a model for ZFC.

We now recall some terminology.

Definition 2.1. (1) A set or cardinal is $\mathcal{U}$-small if it has the same cardinality as an element of $\mathcal{U}$.

(2) A category $\mathcal{C}$ consists of a set(!) of "objects" $\mathrm{Ob}(\mathcal{C})$ and a set of "arrows" $\operatorname{Mor}(\mathcal{C})$ with the usual extra structure.

(3) $\mathcal{U}$-Set is the category whose objects consist of elements of $\mathcal{U}$ and whose Hom-sets are just the standard Hom's between sets. Likewise, if $\mathcal{E}$ is a "structure" [5] (e.g. abelian groups or rings), then $\mathcal{U}-\mathcal{E}$ is the category of $\mathcal{E}$-objects whose underlying set is in $\mathcal{U}$. In particular, $\mathcal{U}-$ Cat (resp. $\mathcal{U}-\mathrm{Gd}$ ) is the category of categories (resp. groupoids) $\mathcal{C}$ with $\mathrm{Ob}(\mathcal{C}) \in \mathcal{U}$ and $\operatorname{Mor}(\mathcal{C}) \in \mathcal{U}$ and the usual Hom's between categories.

(4) A category is $\mathcal{U}$-small if both its objects and arrows are $\mathcal{U}$-small sets.

(5) A category is essentially $\mathcal{U}$-small if it equivalent to a $\mathcal{U}$-small category.

(6) A category is a $\mathcal{U}$-category if it has $\mathcal{U}$-small Hom-sets.

(7) An abelian $\mathcal{U}$-category $\mathcal{C}$ is $\mathcal{U}$-Grothendieck if $\mathcal{C}$ has a generator; $\mathcal{U}$-small colimits exist in $\mathcal{C}$ and $\mathcal{U}$-small filtered colimits are exact.

Remark 2.2. If $E \in \mathrm{Ob}(\mathcal{U}-\mathcal{E})$, then $E \in \mathcal{U}$ since $E$ is described by an element of $\mathcal{U}$. For example, $\mathcal{C} \in \mathrm{Ob}(\mathcal{U}-\mathrm{Cat})$ is described by an element of $\mathcal{U}^{\times 6} \subset \mathcal{U}$.

Lemma 2.3. For $\mathcal{C}, \mathcal{D} \in \mathcal{U}$-Cat, $\operatorname{Hom}(\mathcal{C}, \mathcal{D}) \in \mathcal{U}$-Cat.

Remark 2.4. The axiom of choice allows us to replace a $\mathcal{U}$-category $\mathcal{C}$ with an isomorphic category $\mathcal{C}^{\prime}$, with $\mathrm{Ob}\left(\mathcal{C}^{\prime}\right)=\operatorname{Ob}(\mathcal{C})$ and $\mathcal{C}^{\prime}(C, D) \in \mathcal{U}$ for every $C, D \in$ 
$\mathrm{Ob}(\mathcal{C})$. In particular, if $\mathcal{C}$ is a (pre-additive) $\mathcal{U}$-category, we can define representable functors

$$
\mathcal{C}(C,-): \mathcal{C} \longrightarrow \mathcal{U}-\operatorname{Set}(\mathcal{U}-\mathrm{Ab}): D \longmapsto \mathcal{C}^{\prime}(C, D),
$$

where $\mathcal{C}^{\prime}$ is as above.

The universe axiom is the basis for the very useful "extension of the universe" principle. I.e. by selecting a large enough universe we may assume that any individual category is small. The theory of universes comes at a price however, namely the dependence of the notations on the chosen universe. Since this is rather tedious one usually fixes the universe in advance and then drops it from the notations except when invoking the extension of the universe principle. We will follow these conventions in this paper.

2.2. Some constructions depending on the universe. If $\mathfrak{a}$ is a pre-additive category, then we denote by $\mathcal{U}-\operatorname{Mod}(\mathfrak{a})$ the category of covariant additive functors from $\mathfrak{a}$ to $\mathcal{U}-\mathrm{Ab}$. It is easy to see that if $\mathfrak{a}$ is essentially $\mathcal{U}$-small, then $\mathcal{U}-\operatorname{Mod}(\mathfrak{a})$ is a $\mathcal{U}$-Grothendieck category. If $\mathfrak{a}$ is a $\mathcal{U}$-category, $\mathcal{U}-\operatorname{Mod}(\mathfrak{a})$ contains functors $\mathfrak{a}(A,-): \mathfrak{a} \longrightarrow \mathcal{U}-\mathrm{Ab}$ for $A \in \mathfrak{a}$ (see Remark 2.4). In this case we define $\mathcal{U}-$ $\bmod (\mathfrak{a})$ as the full subcategory of $\mathcal{U}-\operatorname{Mod}(\mathfrak{a})$ containing all functors that can be written as cokernels of maps $\bigoplus_{i=1}^{m} \mathfrak{a}\left(A_{i},-\right) \longrightarrow \bigoplus_{j=1}^{n} \mathfrak{a}\left(B_{j},-\right)$. If $\mathcal{U} \subset \mathcal{V}$ is an inclusion of universes, then Yoneda's lemma yields an equivalence of categories $\mathcal{U}-$ $\bmod (\mathfrak{a}) \longrightarrow \mathcal{V}-\bmod (\mathfrak{a})$.

If $\mathcal{C}$ is an essentially small $\mathcal{U}$-category, then $\mathcal{U}-\operatorname{Ind}(\mathcal{C})$ is the full subcategory of $\mathcal{U}-\operatorname{Mod}(\mathcal{C})$ consisting of left exact functors. It is well known that $\mathcal{U}-\operatorname{Ind}(\mathcal{C})$ is a $\mathcal{U}$ Grothendieck category. The objects in $\mathcal{U}-\operatorname{Ind}(\mathcal{C})$ may be written as formal $\mathcal{U}$-small filtered colimits of objects in $\mathcal{C}$, and the Hom-sets are computed by the rule

$$
\operatorname{Hom}_{\mathcal{U}-\operatorname{Ind}(\mathcal{C})}\left(\operatorname{colim}_{i \in I} A_{i}, \operatorname{colim}_{j \in J} B_{j}\right)=\lim _{i \in I} \operatorname{colim}_{j \in J} \operatorname{Hom}_{\mathcal{C}}\left(A_{i}, B_{j}\right) .
$$

An object $C$ in a $\mathcal{U}$-category $\mathcal{C}$ is $\mathcal{U}$-finitely presented if the functor $\mathcal{C}(C,-)$ : $\mathcal{C} \longrightarrow \mathcal{U}$-Set (see Remark 2.4) preserves $\mathcal{U}$-small filtered colimits. We define $\mathcal{U}-\operatorname{Fp}(\mathcal{C})$ as the full subcategory of $\mathcal{C}$ containing precisely the $\mathcal{U}$-finitely presented objects. It is well known that if $\mathcal{C}$ contains a $\mathcal{U}$-small full subcategory $\mathfrak{g}$ of $\mathcal{U}$-finitely presented generators of $\mathcal{C}$, then $\mathcal{U}-\operatorname{Fp}(\mathcal{C})$ is the finite colimit closure of $\mathfrak{g}$ in $\mathcal{C}$ [4] and in particular is essentially $\mathcal{U}$-small. If $\mathfrak{a}$ is essentially $\mathcal{U}$-small, it is well known and easy to see that $\mathcal{U}-\operatorname{Fp}(\mathcal{U}-\operatorname{Mod}(\mathfrak{a}))=\mathcal{U}-\bmod (\mathfrak{a})$.

A $\mathcal{U}$-Grothendieck category $\mathcal{C}$ is locally coherent if it has a $\mathcal{U}$-small set of $\mathcal{U}$ finitely presented generators and $\mathcal{U}-\mathrm{Fp}(\mathcal{C})$ is a (necessarily essentially $\mathcal{U}$-small) abelian category.

If $\mathcal{C}$ is a locally coherent $\mathcal{U}$-Grothendieck category, then the natural functor

$$
\mathcal{U}-\operatorname{Ind}(\mathcal{U}-\mathrm{Fp}(\mathcal{C})) \longrightarrow \mathcal{C}
$$

is an equivalence of categories. If $\mathcal{C}$ is essentially $\mathcal{U}$-small, then the natural functor

$$
\mathcal{C} \longrightarrow \mathcal{U}-\operatorname{Fp}(\mathcal{U}-\operatorname{Ind}(\mathcal{C}))
$$

is an equivalence as well.

Convention. From now on we work with a fixed universe $\mathcal{U}$. All categories will be $\mathcal{U}$-categories. The notions of small and essentially small are with respect to $\mathcal{U}$. The same holds for the notion of a Grothendieck category. The symbols Mod, Ind, Fp 
and mod are implicitly prefixed by $\mathcal{U}$. Individual objects such as abelian groups, rings, and modules are, unless otherwise specified, assumed to be $\mathcal{U}$-small.

2.3. $R$-linear abelian categories. Consider a commutative ring $R$. Recall that an $R$-linear category is a pre-additive category $\mathfrak{a}$ together with a ring map $\rho: R \rightarrow$ $\operatorname{Nat}\left(1_{\mathfrak{a}}, 1_{\mathfrak{a}}\right) . \rho$ induces a ring map $\rho_{A}: R \longrightarrow \mathfrak{a}(A, A)$ for every object $A$ and an action of $R$ on every Hom-set. This leads to the equivalent definition of an $R$ linear category as a category enriched in the category $\operatorname{Mod}(R)$ of $R$-modules. A pre-additive category is of course the same as a $\mathbb{Z}$-linear category.

Unless otherwise stated, we will assume the ring $R$ to be coherent. Let $\bmod (R)$ denote the full abelian subcategory of $\operatorname{Mod}(R)$ of finitely presented $R$-modules. Consider an abelian $R$-linear category $\mathcal{C}$. For every object $C$ of $\mathcal{C}$ we obtain a (up to a canonical natural isomorphism) unique finite colimit preserving functor

$$
\left(-\otimes_{R} C\right): \bmod (R) \longrightarrow \mathcal{C}
$$

with $R \otimes_{R} C=C$. We can construct its left derived functors $\operatorname{Tor}_{i}^{R}(-, C)$ using projective resolutions in $\bmod (R)$. In fact, we naturally have ( $R$-bilinear) bifunctors

$$
\operatorname{Tor}_{i}^{R}(-,-): \bmod (R) \times \mathcal{C} \longrightarrow \mathcal{C} .
$$

The functors $\left(X \otimes_{R}-\right)$ are finite colimit preserving. It is easily seen (using a fixed free resolution of $X)$ that the functors $\operatorname{Tor}_{i}^{R}(X,-)$ form a homological $\delta$-functor.

In a completely analogous way we define

$$
\operatorname{Hom}_{R}(-, C):(\bmod (R))^{\mathrm{op}} \longrightarrow \mathcal{C}
$$

as the unique finite limit-preserving functor with $\operatorname{Hom}_{R}(R, C)=C$, and taking its right derived functors (again using projective resolutions in $\bmod (R)$ ) we obtain ( $R$-bilinear) bifunctors

$$
\operatorname{Ext}_{R}^{i}(-,-):(\bmod (R))^{\mathrm{op}} \times \mathcal{C} \longrightarrow \mathcal{C} .
$$

The functors $\operatorname{Hom}_{R}(X,-)$ are finite limit preserving. The functors $\operatorname{Ext}_{R}^{i}(X,-)$ form a cohomological $\delta$-functor.

Note that $\operatorname{Hom}_{R}(X,-): \mathcal{C} \longrightarrow \mathcal{C}$ is in fact nothing but the opposite of the tensor functor $\left(X \otimes_{R}-\right): \mathcal{C}^{\mathrm{op}} \longrightarrow \mathcal{C}^{\mathrm{op}}$ of $\mathcal{C}^{\mathrm{op}}$.

Definition 2.5 ([3]). An object $C$ of $\mathcal{C}$ is flat (over $R$ ) if the functor $\left(-\otimes_{R} C\right)$ : $\bmod (R) \longrightarrow \mathcal{C}$ is exact. Dually, $C$ is coflat (over $R$ ) if $\operatorname{Hom}_{R}(-, C): \bmod (R) \longrightarrow \mathcal{C}$ is exact.

Remark 2.6. Flatness of $C$ is equivalent to the vanishing of $\operatorname{Tor}_{1}^{R}(-, C)$ and to the vanishing of all $\operatorname{Tor}_{i}^{R}(-, C)$ for $i \geq 1$. Dually, coflatness of $C$ is equivalent to the vanishing of $\operatorname{Ext}_{R}^{1}(-, C)$ and to the vanishing of all $\operatorname{Ext}_{R}^{i}(-, C)$ for $i \geq 1$. Consequently, in an exact sequence $0 \longrightarrow A \longrightarrow B \longrightarrow C \longrightarrow 0$, if $B$ and $C$ are flat, the same holds for $A$, and dually, if $A$ and $B$ are coflat, the same holds for $C$.

We will now list some useful facts concerning the $\operatorname{Hom}_{R}$ and $\otimes_{R}$ functors.

Proposition 2.7. Consider an R-linear functor $F: \mathcal{C} \longrightarrow \mathcal{D}$ between abelian $R$ linear categories. If $F$ is exact, then for $X \in \bmod (R)$ and $C \in \mathcal{C}$

$$
\operatorname{Ext}_{R}^{i}(X, F(C))=F\left(\operatorname{Ext}_{R}^{i}(X, C)\right) \text {. }
$$

If $F$ is only left exact, then (2.2) holds for $i=0$.

Proof. This follows if we replace $X$ by a free resolution. 
Corollary 2.8. Consider an R-linear category $\mathcal{C}$ and a small category $\mathcal{I}$. If $\mathcal{I}$ colimits are exact in $\mathcal{C}, \operatorname{Ext}_{R}^{i}(X,-)$ preserves them for $X \in \bmod (R)$.

Proof. By taking $F$ to be the functor colim : $\operatorname{Fun}(\mathcal{I}, \mathcal{C}) \longrightarrow \mathcal{C}$, this follows from Proposition 2.7.

Proposition 2.9. For $X, Y \in \bmod (R)$ and $C, D, E \in \mathcal{C}$, the following hold:

(0) $\mathcal{C}\left(X \otimes_{R} C, D\right) \cong \operatorname{Hom}_{R}(X, \mathcal{C}(C, D)) \cong \mathcal{C}\left(C, \operatorname{Hom}_{R}(X, D)\right)$;

(1) $\operatorname{Hom}_{R}\left(X \otimes_{R} Y, C\right) \cong \operatorname{Hom}_{R}\left(X, \operatorname{Hom}_{R}(Y, C)\right)$;

(2) $\left(X \otimes_{R} Y\right) \otimes_{R} C \cong X \otimes_{R}\left(Y \otimes_{R} C\right)$;

(3) if $C$ is coflat, $X \otimes_{R} \operatorname{Hom}_{R}(Y, C) \cong \operatorname{Hom}_{R}\left(\operatorname{Hom}_{R}(X, Y), C\right)$;

(4) if $D$ is flat, $\operatorname{Hom}_{R}\left(X, Y \otimes_{R} D\right) \cong \operatorname{Hom}_{R}(X, Y) \otimes_{R} D$;

(5) if $E$ is injective, $X \otimes_{R} \mathcal{C}(C, E) \cong \mathcal{C}\left(\operatorname{Hom}_{R}(X, C), E\right)$;

(6) if $E$ is injective and $C$ is coflat, then $\mathcal{C}(C, E)$ is flat;

(7) if $E$ is injective and $C$ is flat, then $\mathcal{C}(C, E)$ is coflat;

(8) if $\operatorname{Ext}^{1}\left(C, Z \otimes_{R} E\right)=0$ for all $Z$ in $\bmod (R)$ and $E$ is flat, then $X \otimes_{R}$ $\mathcal{C}(C, E)=\mathcal{C}\left(C, X \otimes_{R} E\right)$ and $\mathcal{C}(C, E)$ is flat.

Consider a finitely generated ideal $I$ of $R$. For every $C \in \mathcal{C}$, we obtain a map $I \otimes_{R} C \longrightarrow C$, which is not necessarily mono unless $C$ is flat. We will denote the image of this map by $I C$. Dually, we will denote the image of the map $C \longrightarrow$ $\operatorname{Hom}_{R}(I, C)$ by $C I$. It is easy to see that $(I J) C=I(J C)$ as subobjects of $C$.

If $(\mathfrak{a}, \rho)$ is an $R$-linear category and $F \in \operatorname{Mod}(\mathfrak{a})$, then the abelian groups $F(A)$ inherit an $R$-module structure from the maps $\rho(r): A \longrightarrow A$. In fact, $\operatorname{Mod}(\mathfrak{a})$ is isomorphic to the $R$-linear category of $R$-linear functors from $\mathfrak{a}$ into $\operatorname{Mod}(R)$ (see also 4 (4).

Proposition 2.10. For an essentially small R-linear category $\mathfrak{a}$, the $\operatorname{Hom}_{R}$ and $\otimes_{R}$ functors on $\operatorname{Mod}(\mathfrak{a})$ are computed pointwise. I.e.

$$
\begin{aligned}
\left(X \otimes_{R} F\right)(A) & =X \otimes_{R} F(A), \\
\operatorname{Hom}_{R}(X, F)(A) & =\operatorname{Hom}_{R}(X, F(A))
\end{aligned}
$$

for $X \in \bmod (R), F \in \operatorname{Mod}(\mathfrak{a})$ and $A \in \mathfrak{a}$. In particular, $F$ in $\operatorname{Mod}(\mathfrak{a})$ is flat (resp. coflat) if and only if all its values $F(A)$ are flat (resp. coflat) in $\operatorname{Mod}(R)$.

2.4. Derived functors and ind-objects. Some arguments in this paper are based on extending derived functors to ind-objects (see \$2.2). Therefore in this section we discuss some of the relevant properties in this regard.

Definition 2.11. (1) A functor $F: \mathcal{A} \longrightarrow \mathcal{B}$ between an arbitrary category $\mathcal{A}$ and a pre-additive category $\mathcal{B}$ is called effaceable 11 if for every $A \in \mathcal{A}$ there is a monomorphism $u: A \longrightarrow A^{\prime}$ with $F(u)=0 . \quad F$ is called coeffaceable if for every $A$ there is an epimorphism $u: A^{\prime} \longrightarrow A$ with $F(u)=$ 0.

(2) A functor $F: \mathcal{A} \longrightarrow$ Ab is called weakly effaceable if for each $A \in \mathcal{A}$ and $x \in F(A)$ there is a monomorphism $u: A \longrightarrow A^{\prime}$ with $F(u)(x)=0$.

Proposition 2.12 ([11). Let $F: \mathcal{A} \longrightarrow \mathcal{B}$ be an additive functor between abelian categories. If $\mathcal{A}$ has enough injectives, then the following are equivalent:

(1) $F$ is effaceable;

(2) $F(I)=0$ for all injectives $I$ of $\mathcal{A}$. 
Consider an extension to ind-objects

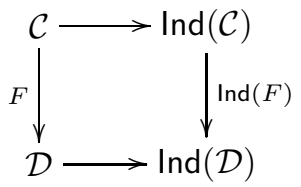

where $\mathcal{C}, \mathcal{D}$ are essentially small abelian categories.

Proposition 2.13. The following are equivalent:

(1) $F$ is effaceable;

(2) $\operatorname{Ind}(F)$ is effaceable.

Proof. Suppose $\operatorname{Ind}(F)$ is effaceable and consider $C$ in $\mathcal{C}$. There is a monomorphism $u: C \longrightarrow \operatorname{colim}_{i} C_{i}$ in $\operatorname{Ind}(\mathcal{C})$ with $\operatorname{Ind}(F)(u)=0$ for certain $C_{i}$ in $\mathcal{C}$. Consider the maps $s_{j}: C_{j} \longrightarrow \operatorname{colim}_{i} C_{i}$. Since $C$ is finitely presented, $u$ factorizes as $u=s_{j} \circ f$ for some $f: C \longrightarrow C_{j}$. Now $s_{j}$ is mapped by $\operatorname{lnd}(F)$ onto the canonical map $F\left(C_{j}\right) \longrightarrow$ $\operatorname{colim}_{i} F\left(C_{i}\right)$, and its composition with $F(f)$ is zero. Since $F(C)$ is finitely presented in $\operatorname{Ind}(\mathcal{D})$, there is a map $s_{j k}: C_{j} \longrightarrow C_{k}$ such that $F\left(s_{j k}\right) \circ F(f)=0$, so $s_{j k} \circ f$ is a monomorphism that serves our purpose.

Now suppose $F$ is effaceable. By Proposition 2.12 it suffices to prove that $\operatorname{Ind}(F)(I)=0$ for every injective $I=\operatorname{colim}_{i} C_{i}$ of $\operatorname{Ind}(\mathcal{C})$. For every $C_{i}$, we take a monomorphism $u_{i}: C_{i} \longrightarrow C_{i}^{\prime}$ in $\mathcal{C}$ with $F\left(u_{i}\right)=0$. Since $I$ is injective, the maps $s_{j}: C_{j} \longrightarrow I$ factorize as $s_{j}=f_{j} \circ u_{j}$. It follows that all the maps $\operatorname{Ind}(F)\left(s_{j}\right)$ : $F\left(C_{j}\right) \longrightarrow \operatorname{colim}_{i} F\left(C_{i}\right)$ are zero and hence $\operatorname{Ind}(F)(I)=\operatorname{colim}_{i} F\left(C_{i}\right)=0$.

If $\mathcal{C}$ is an abelian category, then $\operatorname{Ext}_{\mathcal{C}}^{i}(-,-)$ denotes the Yoneda Ext-groups between $\mathcal{C}$-objects. If $\mathcal{C}$ has enough injectives, then $\operatorname{Ext}_{\mathcal{C}}^{i}(C,-)_{i}$ are the derived functors of $\mathcal{C}(C,-)$. The following proposition is presumably well known, but we have been unable to find a reference.

Proposition 2.14. Assume that $\mathcal{C}$ is essentially small. For $A, B \in \mathcal{C}$, we have

$$
\operatorname{Ext}_{\mathcal{C}}^{i}(A, B)=\operatorname{Ext}_{\operatorname{lnd}(\mathcal{C})}^{i}(A, B)
$$

Proof. The formula (2.3) is obviously true for $i=0$. Then by [12, Lemma II.2.1.3], it is sufficient to show that the functor $\operatorname{Ext}_{\operatorname{lnd}(\mathcal{C})}^{i}(-, B)$ restricted to $\mathcal{C}$ is weakly effaceable. This follows from Proposition 2.15 below with $G=\operatorname{Ext}_{\operatorname{lnd}(\mathcal{C})}^{i}(-, B)$.

Proposition 2.15. Assume that $\mathcal{C}$ is essentially small. If in the following commutative diagram

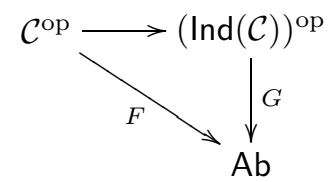

$G$ is weakly effaceable, the same holds for $F$.

Proof. Consider $C$ in $\mathcal{C}$ and $x \in F(C)$. There is an epimorphism $f: \operatorname{colim}_{i} C_{i} \longrightarrow C$ in $\operatorname{Ind}(\mathcal{C})$ with $G(f)(x)=0$ for certain $C_{i}$ in $\mathcal{C}$. Consider the maps $s_{j}: C_{j} \longrightarrow$ $\operatorname{colim}_{i} C_{i}$. We have that $C=\sum_{i} \operatorname{Im}\left(f \circ s_{i}\right)$, but since $C$ is finitely generated in $\operatorname{Ind}(\mathcal{C})$, we find an epimorphism $f \circ s_{j}: C_{j} \longrightarrow C$ in $\operatorname{Ind}(\mathcal{C})$ (and thus in $\mathcal{C}$ ) with $F\left(f \circ s_{j}\right)(x)=G\left(f \circ s_{j}\right)(x)=G\left(s_{j}\right)(G(f)(x))=0$. 


\section{Flatness For $R$-LineAR ABELiAn CATEGORIES}

An $R$-linear category is called flat if its Hom-sets are flat in $\operatorname{Mod}(R)$. In this section, we introduce a different notion of flatness for an $R$-linear abelian category $\mathcal{C}$. This notion has the following properties:

(1) $\mathcal{C}$ is flat if and only if $\mathcal{C}^{\text {op }}$ is flat;

(2) if $\mathcal{C}$ has enough injectives, $\mathcal{C}$ is flat if and only if its injectives are coflat;

(3) if $\mathcal{C}$ is essentially small, $\mathcal{C}$ is flat if and only if $\operatorname{lnd}(\mathcal{C})$ is flat;

(4) if $\mathfrak{a}$ is an essentially small $R$-linear category, $\mathfrak{a}$ is flat (as an $R$-linear category) if and only if $\operatorname{Mod}(\mathfrak{a})$ is flat (as an abelian $R$-linear category).

By enlarging the universe (see \$2.1) we may assume that any category is small. Therefore, in principle, we could take properties (3) and (4) as the definition of flatness. However this would make the self duality (property (2)) very obscure. Therefore we give a somewhat more technical, but manifestly left-right symmetric definition below.

Let $\mathcal{C}$ be an $R$-linear abelian category. The following result is crucial for what follows.

Proposition 3.1. For $X \in \bmod (R)$, the following are equivalent:

(1) $\operatorname{Ext}_{R}^{1}(X,-): \mathcal{C} \longrightarrow \mathcal{C}$ is effaceable;

(2) $\operatorname{Ext}_{R}^{1}(X, \mathcal{C}(-,-)): \mathcal{C}^{\mathrm{op}} \times \mathcal{C} \longrightarrow \mathrm{Ab}$ is weakly effaceable;

(3) $\operatorname{Tor}_{1}^{R}(X,-): \mathcal{C} \longrightarrow \mathcal{C}$ is co-effaceable.

Proof. Since (2) is clearly self-dual, and (3) is the dual statement of (1), it suffices to prove the equivalence of (1) and (2). Take a projective resolution $P$. of $X$ and let $Y$ denote the kernel of $P_{0} \longrightarrow X$. Note that since

$$
\operatorname{Ext}^{1}(X, \mathcal{C}(A, B))=H_{1} \mathcal{C}\left(A, \operatorname{Hom}_{R}(P ., B)\right),
$$

an element in this group can be represented by a $\mathcal{C}$-map $A \longrightarrow \operatorname{Hom}_{R}\left(P_{1}, B\right)$ such that the composition with $\operatorname{Hom}_{R}\left(P_{1}, B\right) \longrightarrow \operatorname{Hom}_{R}\left(P_{2}, B\right)$ is zero. Suppose (2) holds. Consider an arbitrary $\mathcal{C}$-map $A \longrightarrow \operatorname{Ext}_{R}^{1}(X, B)$. We will show that there is a mono $B \longrightarrow B^{\prime}$ such that $A \longrightarrow \operatorname{Ext}_{R}^{1}(X, B) \longrightarrow \operatorname{Ext}_{R}^{1}\left(X, B^{\prime}\right)$ is zero, which proves (1). Consider the pullback

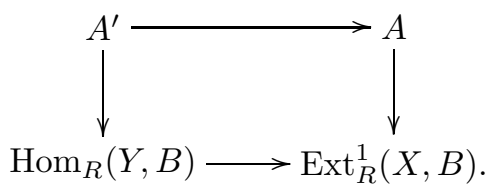

The composition $A^{\prime} \longrightarrow \operatorname{Hom}_{R}(Y, B) \longrightarrow \operatorname{Hom}_{R}\left(P_{1}, B\right)$ represents an element of $\operatorname{Ext}^{1}(X, \mathcal{C}(A, B))$, hence by $(2)$ we can take $A^{\prime \prime} \longrightarrow A^{\prime}$ and $B \longrightarrow B^{\prime}$ such that $A^{\prime \prime} \longrightarrow A^{\prime} \longrightarrow \operatorname{Hom}_{R}(Y, B) \longrightarrow \operatorname{Hom}_{R}\left(Y, B^{\prime}\right)$ factors over $\operatorname{Hom}_{R}\left(P_{0}, B^{\prime}\right) \longrightarrow$ $\operatorname{Hom}_{R}\left(Y, B^{\prime}\right)$. It follows that the composition $A^{\prime \prime} \longrightarrow A^{\prime} \longrightarrow \operatorname{Hom}_{R}(Y, B) \longrightarrow$ $\operatorname{Hom}_{R}\left(Y, B^{\prime}\right) \longrightarrow \operatorname{Ext}_{R}^{1}\left(X, B^{\prime}\right)$ is zero, and consequently $A \longrightarrow \operatorname{Ext}_{R}^{1}(X, B) \longrightarrow$ $\operatorname{Ext}_{R}^{1}\left(X, B^{\prime}\right)$ is zero, too.

Now suppose (1) holds, and consider an element in $\operatorname{Ext}^{1}(X, \mathcal{C}(A, B))$ represented by $A \longrightarrow \operatorname{Hom}_{R}\left(P_{1}, B\right)$. Take a monomorphism $B \longrightarrow B^{\prime}$ such that $\operatorname{Ext}_{R}^{1}(X, B) \longrightarrow \operatorname{Ext}_{R}^{1}\left(X, B^{\prime}\right)$ is zero. Let $K$ denote the kernel of $\operatorname{Hom}_{R}\left(P_{1}, B\right) \longrightarrow$ $\operatorname{Hom}_{R}\left(P_{2}, B\right)$ and let $L^{\prime}$ denote the image of $\operatorname{Hom}_{R}\left(P_{0}, B^{\prime}\right) \longrightarrow \operatorname{Hom}_{R}\left(P_{1}, B^{\prime}\right)$. We 
obtain the following factorization:

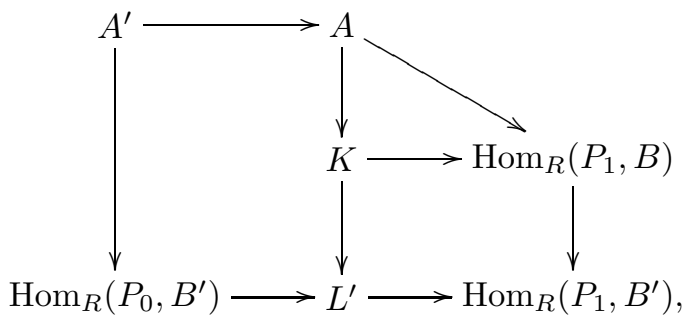

in which $A^{\prime}$ is defined as a pullback. Clearly, the composition $A^{\prime} \longrightarrow \operatorname{Hom}_{R}\left(P_{1}, B^{\prime}\right)$ represents zero in $\operatorname{Ext}^{1}\left(X, \mathcal{C}\left(A^{\prime}, B^{\prime}\right)\right)$.

Definition 3.2. The category $\mathcal{C}$ is called flat (over $R$ ) if every $X \in \bmod (R)$ satisfies the equivalent conditions of Proposition 3.1 .

Note that the notion of flatness is independent of the choice of universe.

Proposition 3.3. The following are equivalent:

(1) $\mathcal{C}$ is flat over $R$;

(2) $\mathcal{C}^{\text {op }}$ is flat over $R$.

Proof. Immediate from Proposition 3.1 .

Proposition 3.4. If $\mathcal{C}$ has enough injectives, the following are equivalent:

(1) $\mathcal{C}$ is flat over $R$;

(2) injectives in $\mathcal{C}$ are coflat.

Proof. This follows from Proposition 2.12 with $F=\operatorname{Ext}_{R}^{1}(X,-)$.

Proposition 3.5. If $\mathcal{C}$ is flat (over $R$ ), the following hold:

(1) for every $X \in \bmod (R)$ and $i \geq 1, \operatorname{Ext}_{R}^{i}(X,-)$ is effaceable;

(2) for every $X \in \bmod (R)$, the cohomological $\delta$-functor $\left(\operatorname{Ext}_{R}^{i}(X,-)\right)_{i \geq 0}$ is universal [11];

(3) for every $X \in \bmod (R)$ and $i \geq 1, \operatorname{Tor}_{i}^{R}(X,-)$ is co-effaceable.

(4) for every $X \in \bmod (R)$, the homological $\delta$-functor $\left(\operatorname{Tor}_{i}^{R}(X,-)\right)_{i \geq 0}$ is universal.

Proof. (1) and (3) follow by dimension shifting in the first argument. (2) and (4) follow from (1) and (3) by [11.

The category of ind-objects $\operatorname{Ind}(\mathcal{C})$ (see $\$ 2.2$ ) is obviously $R$-linear. It follows from Proposition 2.7 that the functor $\mathcal{C} \longrightarrow \operatorname{Ind}(\mathcal{C})$ commutes with $\operatorname{Tor}^{R}$ and $\operatorname{Ext}_{R}$. So $\operatorname{Tor}_{i}^{\operatorname{Ind}(\mathcal{C}), R}(X,-)$ and $\operatorname{Ext}_{\operatorname{lnd}(\mathcal{C}), R}^{i}(X,-)$ are the extensions to ind-objects of $\operatorname{Tor}_{i}^{\mathcal{C}, R}(X,-)$ and $\operatorname{Ext}_{\mathcal{C}, R}^{i}(X,-)$.

Proposition 3.6. Assume that $\mathcal{C}$ is essentially small. The following are equivalent:

(1) $\mathcal{C}$ is flat over $R$;

(2) $\operatorname{Ind}(\mathcal{C})$ is flat over $R$.

Proof. This follows immediately from Proposition 2.13 with $F=\operatorname{Ext}_{\mathcal{C}, R}^{1}(X,-)$ and $\operatorname{lnd}(F)=\operatorname{Ext}_{\operatorname{lnd}(\mathcal{C}), R}^{1}(X,-)$. 
Proposition 3.7. For an essentially small R-linear category $\mathfrak{a}$, the following are equivalent:

(1) $\mathfrak{a}$ is flat over $R$;

(2) the abelian category $\operatorname{Mod}(\mathfrak{a})$ is flat over $R$.

Proof. First, note that for every $E$ in $\operatorname{Mod}(\mathfrak{a})$ and for every $A$ in $\mathfrak{a}$,

$$
\begin{aligned}
\operatorname{Mod}(R)(X, E(A)) & =\operatorname{Mod}(R)(X, \operatorname{Mod}(\mathfrak{a})(\mathfrak{a}(A,-), E)) \\
& =\operatorname{Mod}(\mathfrak{a})\left(X \otimes_{R} \mathfrak{a}(A,-), E\right) .
\end{aligned}
$$

By Proposition 2.10, $\operatorname{Mod}(\mathfrak{a})$ is flat if and only if for every injective $E$ in $\operatorname{Mod}(\mathfrak{a})$ and every $A$ in $\mathfrak{a}, E(A)$ is coflat. Also by Proposition 2.10, $\mathfrak{a}$ is flat if and only if every functor $\mathfrak{a}(A,-)$ for $A$ in $\mathfrak{a}$ is flat. Also, since $\operatorname{Mod}(\mathfrak{a})$ has enough injectives, this is equivalent to requiring that for every injective $E$ in $\operatorname{Mod}(\mathfrak{a})$ the functor $\operatorname{Mod}(\mathfrak{a})(-, E) \circ\left(-\otimes_{R} \mathfrak{a}(A,-)\right): \bmod (R) \longrightarrow \operatorname{Mod}(R)$ is exact. The statement then follows from the computation above.

\section{BASE CHANGE}

In this section we study a natural substitute for the notion of base change in algebraic geometry, and we show that it is compatible with constructions such as Ind and Mod.

We fix a homomorphism of commutative rings $\theta: R \longrightarrow S$. For $M \in \operatorname{Mod}(S)$, $\bar{M}$ denotes $M$ considered as an $R$-module using $\theta$. For an $S$-linear category $\mathfrak{b}, \overline{\mathfrak{b}}$ is the $R$-linear category with $\mathrm{Ob}(\overline{\mathfrak{b}})=\mathrm{Ob}(\mathfrak{b})$ and $\overline{\mathfrak{b}}\left(B, B^{\prime}\right)=\overline{\mathfrak{b}\left(B, B^{\prime}\right)}$. More often than not we will just write $\mathfrak{b}$ for $\overline{\mathfrak{b}}$.

For an $R$-linear category $\mathfrak{a}, S \otimes_{R} \mathfrak{a}$ is the $S$-linear category with $\operatorname{Ob}\left(S \otimes_{R} \mathfrak{a}\right)=$ $\mathrm{Ob}(\mathfrak{a})$ and $\left(S \otimes_{R} \mathfrak{a}\right)\left(A, A^{\prime}\right)=S \otimes_{R} \mathfrak{a}\left(A, A^{\prime}\right)$.

The functor $S \otimes_{R}(-)$ is left adjoint to $\overline{(-)}$ in the sense that for an $R$-linear category $\mathfrak{a}$ and an $S$-linear category $\mathfrak{b}$, there is an isomorphism, natural in $\mathfrak{a}, \mathfrak{b}$ :

$$
\overline{\operatorname{Add}(S)\left(S \otimes_{R} \mathfrak{a}, \mathfrak{b}\right)} \cong \operatorname{Add}(T)(\mathfrak{a}, \overline{\mathfrak{b}})
$$

as $R$-linear categories where $\operatorname{Add}(T)$ denotes the $T$-linear functors.

For an $R$-linear category $(\mathcal{C}, \rho)$, let $\mathcal{C}_{S}$ be the following $S$-linear category of $S$-objects: the objects of $\mathcal{C}_{S}$ are couples $(C, \varphi)$, where $C$ is an object of $\mathcal{C}$ and $\varphi: S \longrightarrow \mathcal{C}(C, C)$ is a ring map with $\varphi \circ \theta=\rho_{C}$. The morphisms of $\mathcal{C}_{S}$ are the obvious compatible $\mathcal{C}$-morphisms. Clearly $\mathcal{C}_{S}$ becomes $S$-linear using the ring maps $\varphi$. The functor $(-)_{S}$ is right adjoint to $\overline{(-)}$ in the sense that for an $R$-linear category $\mathcal{C}$ and an $S$-linear category $\mathcal{D}$, there is an isomorphism, natural in $\mathcal{C}, \mathcal{D}$ :

$$
\overline{\operatorname{Add}(S)\left(\mathcal{D}, \mathcal{C}_{S}\right)} \cong \operatorname{Add}(R)(\overline{\mathcal{D}}, \mathcal{C})
$$

of $R$-linear categories.

Remark 4.1. It is readily seen that $(\operatorname{Mod}(R))_{S} \cong \operatorname{Mod}(S)$, hence we deduce the isomorphism $\operatorname{Add}(S)(\mathfrak{b}, \operatorname{Mod}(S)) \cong \operatorname{Mod}(\mathfrak{b})$ for an $S$-linear category $\mathfrak{b}$ which was mentioned just before Proposition 2.10 .

Proposition 4.2. The forgetful functor $F: \mathcal{C}_{S} \longrightarrow \mathcal{C}$ preserves, reflects and creates [1] all limits and colimits. 
Proof. We give the construction of limits in $\mathcal{C}_{S}$ that exist in $\mathcal{C}$. Let $G: \mathcal{I} \longrightarrow \mathcal{C}_{S}$ with $G(i)=\left(C_{i}, \varphi_{i}\right)$ be an arbitrary functor and $\operatorname{let} \lim _{i} C_{i}$ denote the limit of $F \circ G$. For every $s \in S$, the maps $\varphi_{i}(s): C_{i} \longrightarrow C_{i}$ define a natural transformation $F \circ G \longrightarrow F \circ G$ mapped by the limit functor to $\lim _{i}\left(\varphi_{i}(s)\right)$. It is easily seen that $\left(\lim _{i} C_{i}, \lim _{i} \varphi_{i}\right)$, where $\left(\lim _{i} \varphi_{i}\right)(s)=\lim _{i}\left(\varphi_{i}(s)\right)$ is a limit of $G$.

When $\mathcal{C}$ is abelian, the same holds for $\mathcal{C}_{S}$, and in this case $\mathcal{C}_{S} \longrightarrow \mathcal{C}$ is exact. From now on we will only use the functor $(-)_{S}$ for abelian categories.

Assume now that $R \longrightarrow S$ is a ring morphism between coherent commutative rings such that $S$ is finitely presented as an $R$-module. The functors $\left(S \otimes_{R}-\right)$ : $\mathcal{C} \longrightarrow \mathcal{C}$ and $\operatorname{Hom}_{R}(S,-): \mathcal{C} \longrightarrow \mathcal{C}$ of $\$ 2.3$ yield functors

$$
\left(S \otimes_{R}-\right): \mathcal{C} \longrightarrow \mathcal{C}_{S}
$$

and

It is easily seen that

$$
\operatorname{Hom}_{R}(S,-): \mathcal{C} \longrightarrow \mathcal{C}_{S}
$$

Proposition 4.3. The functors $\left(S \otimes_{R}-\right)$ and $\operatorname{Hom}_{R}(S,-)$ are, respectively, left and right adjoint to $\mathcal{C}_{S} \longrightarrow \mathcal{C}$. In particular, $\left(S \otimes_{R}-\right)$ preserves projectives and $\operatorname{Hom}_{R}(S,-)$ preserves injectives.

The next result gives a connection between the functors $S \otimes_{R}(-)$ and $(-)_{S}$.

Proposition 4.4. Let $\mathfrak{a}$ be an essentially small R-linear category. Then

(1) there is a commutative diagram

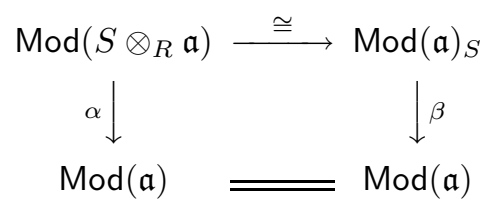

where $\alpha$ is dual to $\mathfrak{a} \longrightarrow S \otimes_{R} \mathfrak{a}$ and $\beta$ is the forgetful functor;

(2) the left and right adjoints to the forgetful functor $\operatorname{Mod}(\mathfrak{a})_{S} \longrightarrow \operatorname{Mod}(\mathfrak{a})$ are computed pointwise, i.e.

$$
\begin{gathered}
\left(S \otimes_{R} F\right)(A)=S \otimes_{R} F A, \\
\operatorname{Hom}_{R}(S, F)(A)=\operatorname{Hom}_{R}(S, F(A)) . \\
\text { In particular } S \otimes_{R} \mathfrak{a}(A,-)=\left(S \otimes_{R} \mathfrak{a}\right)(A,-) .
\end{gathered}
$$

We have the following relation between $\operatorname{Ind}(-)$ and $(-)_{S}$.

Proposition 4.5. Assume that $\mathcal{C}$ is essentially small. The obvious functor

$$
H: \operatorname{lnd}\left(\mathcal{C}_{S}\right) \longrightarrow(\operatorname{Ind}(\mathcal{C}))_{S}
$$

is an equivalence.

Proof. Consider the following commutative diagram with obvious maps:

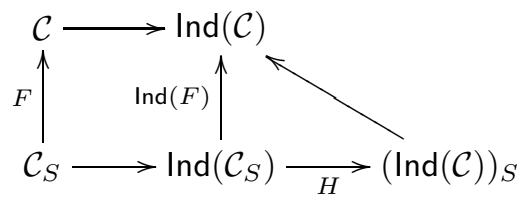

Since $F$ is faithful, the same holds for $\operatorname{lnd}(F)$ and thus for $H$. 
To prove that $H$ is full, consider a map $\left(f_{i}\right)_{i}: \operatorname{colim}_{i}\left(C_{i}, \varphi_{i}\right) \longrightarrow \operatorname{colim}_{j}\left(D_{j}, \psi_{j}\right)$ with $f_{i}: C_{i} \longrightarrow D_{j_{i}}$ maps in $\mathcal{C}$ such that $\left(\psi_{j}\right)_{j} \circ\left(f_{i}\right)_{i}=\left(f_{i}\right)_{i} \circ\left(\varphi_{i}\right)_{i}$ in $\operatorname{lnd}(\mathcal{C})$. This means that for every $i$ we obtain a diagram

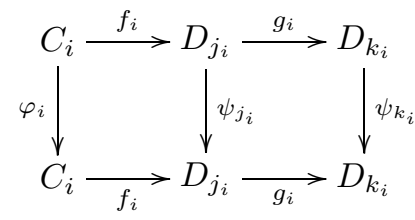

in which the right square commutes and composition with $g_{i}$ makes the left square commute. It follows that the maps $g_{i} \circ f_{i}$ belong to $\mathcal{C}_{S}$ and $\left(f_{i}\right)_{i}=\left(g_{i} \circ f_{i}\right)_{i}$.

To prove that $H$ is essentially surjective, consider an object $C$ of $(\operatorname{Ind}(\mathcal{C}))_{S}$ (we omit the $S$-action $\varphi$ in our notation). $\varphi$ induces an $(\operatorname{Ind}(\mathcal{C}))_{S}$-epimorphism $S \otimes_{R}$ $C \longrightarrow C$ with kernel $K$, for which we obtain another $(\operatorname{Ind}(\mathcal{C}))_{S}$-epimorphism $S \otimes_{R}$ $K \longrightarrow K$. It follows that $C$ is isomorphic to the cokernel of $S \otimes_{R} K \longrightarrow S \otimes_{R} C$. Writing $C=\operatorname{colim}_{i} C_{i}$ as a filtered colimit, we see that $S \otimes_{R} C=\operatorname{colim}_{i}\left(S \otimes_{R} C_{i}\right)$ belongs to $\operatorname{lnd}\left(\mathcal{C}_{S}\right)$ and the same holds for $S \otimes_{R} K$. Since $\operatorname{Ind}(F)$ is exact, the cokernel of the map belongs to $\operatorname{lnd}\left(\mathcal{C}_{S}\right)$, as we wanted.

Next we consider finitely presented objects.

Proposition 4.6. Assume that in $\mathcal{C}$ small filtered colimits are exact. If $(C, \varphi)$ is finitely presented (resp. finitely generated) in $\mathcal{C}_{S}$, then $C$ is finitely presented (resp. finitely generated) in $\mathcal{C}$. The obvious functor

$$
H: \operatorname{Fp}\left(\mathcal{C}_{S}\right) \longrightarrow(\mathrm{Fp}(\mathcal{C}))_{S}
$$

is an isomorphism.

Proof. Consider a finitely presented $(C, \varphi)$ in $\mathcal{C}_{S}$ and a filtered colimit $\operatorname{colim}_{i} C_{i}$ in $\mathcal{C}$. Making use of Corollary 2.8 and the fact that $\mathcal{C}_{S} \longrightarrow \mathcal{C}$ reflects colimits, we may compute

$$
\begin{aligned}
\mathcal{C}\left(C, \operatorname{colim}_{i} C_{i}\right) & =\mathcal{C}_{S}\left((C, \varphi), \operatorname{Hom}_{R}\left(S, \operatorname{colim}_{i} C_{i}\right)\right) \\
& =\mathcal{C}_{S}\left((C, \varphi), \operatorname{colim}_{i} \operatorname{Hom}_{R}\left(S, C_{i}\right)\right) \\
& =\operatorname{colim}_{i} \mathcal{C}_{S}\left((C, \varphi), \operatorname{Hom}_{R}\left(S, C_{i}\right)\right) \\
& =\operatorname{colim}_{i} \mathcal{C}\left(C, C_{i}\right),
\end{aligned}
$$

hence $C$ is finitely presented in $\mathcal{C}$. Now consider the following commutative diagram with obvious maps:

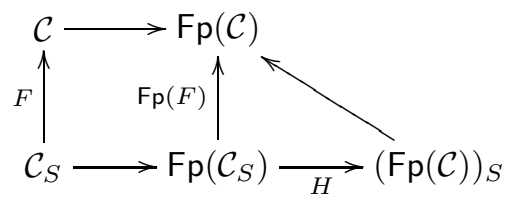

$H$ is readily seen to be fully faithful and injective on objects, so it remains to show that it is surjective on objects. Consider $(C, \varphi)$ in $(\operatorname{Fp}(\mathcal{C}))_{S}$ and a filtered colimit $\operatorname{colim}_{i}\left(C_{i}, \varphi_{i}\right)=\left(\operatorname{colim}_{i} C_{i}, \operatorname{colim}_{i} \varphi_{i}\right)$ in $\mathcal{C}_{S}$. The canonical map

$$
\operatorname{colim}_{i} \mathcal{C}_{S}\left((C, \varphi),\left(C_{i}, \varphi_{i}\right)\right) \longrightarrow \mathcal{C}_{S}\left((C, \varphi),\left(\operatorname{colim}_{i} C_{i}, \operatorname{colim}_{i} \varphi_{i}\right)\right)
$$


is obviously injective. We finish the proof by showing that it is surjective. If $f: C \longrightarrow \operatorname{colim}_{i} C_{i}$ defines a map in the codomain, $f$ factors over some $f_{i}: C \longrightarrow C_{i}$ in $\mathcal{C}$. For each $s \in S$, there is a diagram

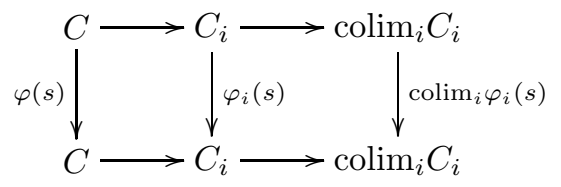

in which the right-hand square is not necessarily commutative. Selecting finitely many generators $s_{k}$ of $S$ over $R$, and using the fact that $C$ is finitely presented in $\mathcal{C}$ and that the colimit is filtered, we can find $C_{i} \longrightarrow C_{j}$ such that the right-hand square with $C_{j}$ instead of $C_{i}$ commutes for every generator $s_{k}$ and hence also for every $s \in S$.

We mention the familiar change of rings spectral sequences.

Proposition 4.7. Let $X \in \bmod (S), C \in \mathcal{C}, A \in \mathcal{C}_{S}$. Assume that $\mathcal{C}$ is flat. There are first quadrant spectral sequences

$$
\begin{aligned}
& E_{2}^{p q}: \operatorname{Ext}_{\mathcal{C}_{S}}^{p}\left(A, \operatorname{Ext}_{R}^{q}(S, C)\right) \Rightarrow \operatorname{Ext}_{\mathcal{C}}^{n}(A, C), \\
& E_{2}^{p q}: \operatorname{Ext}_{S}^{p}\left(X, \operatorname{Ext}_{R}^{q}(S, C)\right) \Rightarrow \operatorname{Ext}_{R}^{n}(X, C) .
\end{aligned}
$$

Proof. By enlarging our universe we may assume that $\mathcal{C}$ is small. Then we replace $\mathcal{C}$ by $\operatorname{lnd}(\mathcal{C})$ in order to have a category with enough injectives. By Proposition 3.6 this enlarged $\mathcal{C}$ is still flat. Furthermore by Proposition 3.5 $\operatorname{Ext}_{R}^{q}(S,-)$ is the derived functor of $\operatorname{Hom}_{R}(S,-)$.

We have

$$
\begin{aligned}
\operatorname{Hom}_{\mathcal{C}_{S}}\left(A, \operatorname{Hom}_{R}(S, C)\right) & =\operatorname{Hom}_{\mathcal{C}}(A, C), \\
\operatorname{Hom}_{S}\left(X, \operatorname{Hom}_{R}(S, C)\right) & =\operatorname{Hom}_{R}(X, C) .
\end{aligned}
$$

Now $\operatorname{Hom}_{R}(S,-)$ preserves injectives and (4.4) shows that $\operatorname{Hom}_{R}(S,-)$ also preserves coflat objects. So $\operatorname{Hom}_{R}(S,-)$ sends injectives to acyclic objects for $\operatorname{Hom}_{\mathcal{C}_{S}}(A,-)$ and $\operatorname{Hom}_{S}(X,-)$.

Thus (4.1), (4.2) are just the Grothendieck spectral sequences [1] associated to (4.3), (4.4).

Here are some properties that are preserved under base change.

Proposition 4.8. $\quad$ (1) If $\mathcal{C}$ has enough injectives, the same holds for $\mathcal{C}_{S}$;

(2) if $\left(G_{i}\right)_{i}$ is a set of generators of $\mathcal{C},\left(S \otimes_{R} G_{i}\right)_{i}$ is a set of generators of $\mathcal{C}_{S}$;

(3) if $\mathcal{C}$ is Grothendieck, the same holds for $\mathcal{C}_{S}$;

(4) if $\mathcal{C}$ is a locally coherent Grothendieck category, the same holds for $\mathcal{C}_{S}$;

(5) if $\mathcal{C}$ is flat over $R$, then $\mathcal{C}_{S}$ is flat over $S$.

Proof. Only (5) is not entirely clear from the above discussion. Consider $C \in \mathcal{C}_{S}$ and $X \in \bmod (S)$. Take a $\mathcal{C}$-monomorphism $m: C \longrightarrow C^{\prime}$ with $\operatorname{Ext}_{R}^{1}(X, m)=0$. Then $\operatorname{Hom}_{R}(S, m): \operatorname{Hom}_{R}(S, C) \longrightarrow \operatorname{Hom}_{R}\left(S, C^{\prime}\right)$ is a monomorphism. The spectral sequence (4.2) yields monomorphism $\operatorname{Ext}_{S}^{1}\left(X, \operatorname{Hom}_{R}(S, C)\right) \longrightarrow \operatorname{Ext}_{S}^{1}(X, C)$ natural in $C$ and hence $\operatorname{Ext}_{S}^{1}\left(X, \operatorname{Hom}_{R}(S, m)\right)=0$. The composition of monomorphims $C \longrightarrow \operatorname{Hom}_{R}(S, C) \longrightarrow \operatorname{Hom}_{R}\left(S, C^{\prime}\right)$ is the required effacing of $\operatorname{Ext}_{S}^{1}(X, C)$ in $\mathcal{C}_{S}$. 


\section{Deformations of ABElian CATEgories}

In this section we introduce deformations of pre-additive and abelian categories. Basically these are lifts along the functors $S \otimes_{R}(-)$ and $(-)_{S}$ for a homomorphism of commutative rings $\theta: R \longrightarrow S$. In the sequel we will assume that $\theta$ is surjective with nilpotent kernel, but this is not necessary for the basic definitions.

We start with the pre-additive case.

Definition 5.1. For an $S$-linear category $\mathfrak{b}$, an $R$-deformation of $\mathfrak{b}$ is an $R$-linear category $\mathfrak{a}$ together with an $R$-linear functor $\mathfrak{a} \longrightarrow \overline{\mathfrak{b}}$ that induces an equivalence $S \otimes_{R} \mathfrak{a} \longrightarrow \mathfrak{b}$. A deformation $\mathfrak{a} \longrightarrow \overline{\mathfrak{b}}$ will often be denoted by $\mathfrak{a} \longrightarrow \mathfrak{b}$ or simply by $\mathfrak{a}$. If $S \otimes_{R} \mathfrak{a} \longrightarrow \mathfrak{b}$ is an isomorphism, the deformation is called strict. If $\mathfrak{a}$ is flat over $R$, the deformation is called flat.

Now we consider the abelian case.

Definition 5.2. For an abelian $S$-linear category $\mathcal{D}$, an $R$-deformation of $\mathcal{D}$ is an abelian $R$-linear category $\mathcal{C}$ together with an $R$-linear functor $\overline{\mathcal{D}} \longrightarrow \mathcal{C}$ that induces an equivalence $\mathcal{D} \longrightarrow \mathcal{C}_{S}$. A deformation $\overline{\mathcal{D}} \longrightarrow \mathcal{C}$ will often be denoted by $\mathcal{D} \longrightarrow \mathcal{C}$ or simply by $\mathcal{C}$. If $\mathcal{D} \longrightarrow \mathcal{C}_{S}$ is an isomorphism, the deformation is called strict. If $\mathcal{C}$ is flat over $R$ (as an abelian category), the deformation is called flat.

Remark 5.3. In case of possible confusion we refer to the deformations of Definition 5.1 as linear deformations and to the deformations of Definition 5.2 as abelian deformations.

We will now assume that $\theta: R \longrightarrow S$ is a homomorphism between coherent commutative rings such that $S$ is a finitely presented $R$-module. Then the left and right adjoint to an abelian deformation $\mathcal{D} \longrightarrow \mathcal{C}$ exist, and we will continue to denote them by $S \otimes_{R}-$ and $\operatorname{Hom}_{R}(S,-)$.

Rephrazing the results of $\S \S 3$, 4 in terms of deformations, we deduce that (flat) deformations are preserved under some natural constructions.

Proposition 5.4. Let $\mathfrak{b}$ be an essentially small $S$-linear category. An essentially small linear $R$-deformation $\mathfrak{a} \longrightarrow \mathfrak{b}$ induces an abelian deformation $\operatorname{Mod}(\mathfrak{b}) \longrightarrow$ $\operatorname{Mod}(\mathfrak{a})$. Moreover flatness of these deformations is equivalent.

Proof. We may assume that $\mathfrak{b}=S \otimes_{R} \mathfrak{a}$. Then the result follows from Propositions 3.7 and 4.4 .

Proposition 5.5. Every abelian $R$-deformation $\mathcal{D} \longrightarrow \mathcal{C}$ between essentially small categories induces an abelian $R$-deformation $\operatorname{Ind}(\mathcal{D}) \longrightarrow \operatorname{Ind}(\mathcal{C})$. Moreover flatness of these deformations is equivalent.

Proof. We may assume $\mathcal{D}=\mathcal{C}_{S}$. Then the result follows from Propositions 3.6] and 4.5 .

Proposition 5.6. A deformation $\mathcal{D} \longrightarrow \mathcal{C}$ of locally coherent Grothendieck categories induces a deformation $\mathrm{Fp}(\mathcal{D}) \longrightarrow \mathrm{Fp}(\mathcal{C})$. Moreover flatness of these deformations is equivalent.

Proof. We may assume $\mathcal{D}=\mathcal{C}_{S}$. Then the result follows from Propositions [3.6] and 4.6 . 


\section{Preservation of Properties under nilpotent DeFormations}

In this chapter we investigate some categorical/homological properties that are preserved under flat, nilpotent (see below) abelian deformation. More precisely, we will show that the following properties of an abelian category $\mathcal{D}$ lift to a flat nilpotent deformation:

(1) $\mathcal{D}$ is essentially small;

(2) $\mathcal{D}$ has enough injectives;

(3) $\mathcal{D}$ is a Grothendieck category;

(4) $\mathcal{D}$ is a locally coherent Grothendieck category.

6.1. Nilpotent deformations. As usual $\theta: R \longrightarrow S$ is a morphism between coherent commutative rings such that $S$ is finitely presented over $R$. In order to lift properties from an $S$-linear (abelian) category to an $R$-deformation, we obviously need some further assumptions on the ring map $\theta$. First of all, we will assume that $\theta$ is surjective. In this case, for an abelian $R$-linear $\mathcal{C}$, the forgetful functor $\mathcal{C}_{S} \longrightarrow \mathcal{C}$ is fully faithful. Put $I=\operatorname{ker} \theta$. The hypotheses imply that $I$ is finitely presented.

Next, we will assume that the kernel of $\theta$ is nilpotent.

Definition 6.1. The $R$-deformations (linear or abelian) are called nilpotent provided the ideal $I$ is nilpotent, i.e. $I^{n}=0$ for some $n$. If $I^{n}=0$ the deformation is called (nilpotent) of order $n$.

Remark 6.2. If $\mathcal{C}$ is a nilpotent deformation of $\mathcal{D}$ (linear or abelian), then clearly there exist categories $\mathcal{D}=\mathcal{C}_{0}, \mathcal{C}_{1}, \ldots, \mathcal{C}_{k}=\mathcal{C}$ for certain $k$ such that $\mathcal{C}_{i+1}$ is a nilpotent deformation of order 2 of $\mathcal{C}_{i}$. If $\mathcal{C}$ is flat, then we may assume that the $\left(\mathcal{C}_{i}\right)_{i}$ are flat as well.

Proposition 6.3 ("Nakayama"). Consider a nilpotent deformation $\mathcal{D} \longrightarrow \mathcal{C}$ and $C \in \mathcal{C}$. If either $S \otimes_{R} C=0$ or $\operatorname{Hom}_{R}(S, C)=0$, then $C=0$.

Proof. If $S \otimes_{R} C=0$, then $C=I C$ (2.3). Since $I$ is nilpotent this implies $C=0$. The case $\operatorname{Hom}_{R}(S, C)=0$ is similar.

Proposition 6.4. Consider a nilpotent $R$-deformation $\mathcal{D} \longrightarrow \mathcal{C}$. The functor $\operatorname{Hom}_{R}(S,-): \mathcal{C} \longrightarrow \mathcal{D}$ reflects monomorphisms.

Proof. This readily follows from Proposition 6.3 using kernels.

6.2. Preservation of size. In this section we temporarily drop the assumption that our categories are automatically $\mathcal{U}$-categories and that the base rings $R, S$ are $\mathcal{U}$-small.

We show that nilpotent deformations behave well with respect to size matters.

Lemma 6.5. For $M \in \operatorname{Mod}(R)$,

$$
|M| \leq \sup \left\{\left|S \otimes_{R} M\right|,|\mathbb{N}|\right\} .
$$

Proof. This follows by considering the $I$-adic filtration on $M$.

For a category $\mathcal{C}$, the skeleton $\operatorname{Sk}(\mathcal{C})$ of $\mathcal{C}$ is the set of all isomorphism classes of $\mathcal{C}$-objects. 
Lemma 6.6. Consider an $S$-linear category $\mathfrak{b}$ and let $f: \mathfrak{a} \longrightarrow \mathfrak{b}$ be a nilpotent $R$-deformation of $\mathfrak{b}$. Suppose $\alpha$ is an infinite cardinal such that $|\operatorname{Sk}(\mathfrak{b})| \leq \alpha$ and for all $B, B^{\prime} \in \mathrm{Ob}(\mathfrak{b})$ one has $\left|\mathfrak{b}\left(B, B^{\prime}\right)\right| \leq \alpha$. Then the analogous property holds for $\mathfrak{a}$.

Proof. This follows from the following observations:

(1) By Lemma 6.5, $\left|\mathfrak{a}\left(A, A^{\prime}\right)\right| \leq \sup \left\{\left|\mathfrak{b}\left(f(A), f\left(A^{\prime}\right)\right)\right|,|\mathbb{N}|\right\}$ for $A, A^{\prime} \in \mathfrak{a}$.

(2) By Proposition A.1. $|\operatorname{Sk}(\mathfrak{a})|=|\operatorname{Sk}(\mathfrak{b})|$.

Proposition 6.7. Suppose $S$ is $\mathcal{U}$-small. Consider an $S$-linear category $\mathfrak{b}$ and let $f: \mathfrak{a} \longrightarrow \mathfrak{b}$ be a nilpotent $R$-deformation of $\mathfrak{b}$. If $\mathfrak{b}$ is a $\mathcal{U}$-category (resp. essentially $\mathcal{U}$-small), the same holds for $\mathfrak{a}$.

Proof. Immediate from (the proof of) Lemma 6.6.

We want to prove a similar result for abelian deformations. We prove an analogue of Lemma 6.6. Note that the result we prove is more general than what we immediately need, but its more general form will be used later.

Lemma 6.8. Suppose $S$ is $\mathcal{U}$-small. Consider a flat deformation $\mathcal{D} \longrightarrow \mathcal{C}$ of abelian categories which is of order two. Let $\mathcal{D}^{\prime}$ be a Serre subcategory of $\mathcal{D}$ (i.e. a full subcategory closed under subquotients and extensions) and let $\mathcal{C}^{\prime}$ be the Serre subcategory of $\mathcal{C}$ generated by $\mathcal{D}^{\prime}$ (i.e. the full subcategory of $\mathcal{C}$ of objects $C$ with $S \otimes_{R} C$ (hence also IC) $\in \mathcal{D}^{\prime}$, see also Proposition 7.5).

Suppose $\alpha$ is an infinite cardinal such that $\left|\operatorname{Sk}\left(\mathcal{D}^{\prime}\right)\right| \leq \alpha$, and for all $D, D^{\prime} \in$ $\operatorname{Ob}\left(\mathcal{D}^{\prime}\right)$ one has $\left|\mathcal{D}\left(D, D^{\prime}\right)\right| \leq \alpha$. Then the analogous property holds for $\mathcal{C}^{\prime}$.

Proof. Choose $C, C^{\prime}$. Filtering $C, C^{\prime}$ by the $I$-adic filtration we immediately deduce

$$
\left|\mathcal{C}\left(C, C^{\prime}\right)\right| \leq\left|\mathcal{D}\left(S \otimes_{R} C, I C^{\prime}\right)\right| \cdot\left|\mathcal{D}\left(S \otimes_{R} C, S \otimes_{R} C^{\prime}\right)\right| .
$$

This implies $\left|\mathcal{C}\left(C, C^{\prime}\right)\right| \leq \alpha$.

Concerning $\left|\operatorname{Sk}\left(\mathcal{C}^{\prime}\right)\right|$, we make the following observations:

(1) An object in $\mathcal{C}^{\prime}$ is up to isomorphism determined by a triple $(A, B, e)$, where $A, B \in \mathrm{Ob}\left(\mathcal{D}^{\prime}\right)$ and $e \in \operatorname{Ext}_{\mathcal{C}}^{1}(A, B)$.

(2) The spectral sequence (4.1) yields the bound

$$
\left.\left|\operatorname{Ext}_{\mathcal{C}}^{1}(A, B)\right| \leq \mid \operatorname{Ext}_{\mathcal{D}}^{1}(A, B)\right)|| \operatorname{Hom}_{\mathcal{D}}\left(A, \operatorname{Ext}_{R}^{1}(S, B)\right) \mid .
$$

(3) An element of $\operatorname{Ext}_{\mathcal{D}}^{1}(A, B)$ may be represented by a triple $(C, u, v)$, where $C \in \mathrm{Ob}\left(\mathcal{D}^{\prime}\right)$ and $u \in \mathcal{D}(A, C), v \in \mathcal{D}(C, B)$ are such that $(u, v)$ constitutes a short exact sequence.

The bound $\left|\operatorname{Sk}\left(\mathcal{C}^{\prime}\right)\right| \leq \alpha$ now easily follows.

Proposition 6.9. Suppose $S$ is $\mathcal{U}$-small. Consider a flat nilpotent deformation $\mathcal{D} \longrightarrow \mathcal{C}$ of abelian categories. If $\mathcal{D}$ is a $\mathcal{U}$-category (resp. an essentially $\mathcal{U}$-small category), then so is $\mathcal{C}$.

Proof. We may assume that the deformation is of order two. The case that $\mathcal{D}$ is essentially $\mathcal{U}$-small follows directly from Lemma 6.8. For the case that $\mathcal{D}$ is a $\mathcal{U}$-category we invoke (6.1) to obtain that every $\left|\mathcal{C}\left(C, C^{\prime}\right)\right|$ is bounded by the cardinality of an element of $\mathcal{U}$. 
6.3. Lifting of objects. An important tool in the study of deformations is the lifting of objects along the functors $\left(S \otimes_{R}-\right)$ and $\operatorname{Hom}_{R}(S,-)$. In this section we state some results on lifting that will be used afterwards. We start with the following definition.

Definition 6.10. Consider a functor $H: \mathcal{C} \longrightarrow \mathcal{D}$ and an object $D$ of $\mathcal{D}$. A lift of $D$ along $H$ is an object $C$ of $\mathcal{C}$ and an isomorphism $D \cong H(C)$. Lifts of $D$, together with the obvious morphisms, constitute a groupoid $L(H, D)$.

Remark 6.11. If $H: \mathcal{C} \longrightarrow \mathcal{D}$ is right adjoint to a functor $F: \mathcal{D} \longrightarrow \mathcal{C}$, a lift of $D$ along $H$ can be represented by a map $F(D) \longrightarrow C$. If $H$ is left adjoint to $G: \mathcal{D} \longrightarrow \mathcal{C}$, a lift of $D$ along $H$ can be represented by a map $C \longrightarrow G(D)$.

Consider a flat deformation $\mathcal{D} \longrightarrow \mathcal{C}$. The restriction $\operatorname{Hom}_{R}^{c f}(S,-)$ of $\operatorname{Hom}_{R}(S,-)$ to coflat objects yields a groupoid

$$
L^{c f}(D)=L\left(\operatorname{Hom}_{R}^{c f}(S,-), D\right)
$$

for every coflat $D$ in $\mathcal{D}$. Analogously, the restriction $\left(S \otimes_{R}^{f}-\right)$ of $\left(S \otimes_{R}-\right)$ to flat objects yields a groupoid

$$
L^{f}(D)=L\left(\left(S \otimes_{R}^{f}-\right), D\right)
$$

for every flat $D$ in $\mathcal{D}$.

We state the following theorem without proof. The theorem is a special case of an obstruction theory for derived lifting, which will be published separately [16].

Theorem 6.12. Consider a flat nilpotent $R$-deformation $\mathcal{D} \longrightarrow \mathcal{C}$ of order 2 and a coflat object $D$ of $\mathcal{D}$. There is an obstruction

$$
o(D) \in \operatorname{Ext}_{\mathcal{D}}^{2}\left(\operatorname{Hom}_{S}(I, D), D\right)
$$

satisfying

$$
L^{c f}(D) \neq \varnothing \Longleftrightarrow o(D)=0
$$

over

$$
\operatorname{Ext}_{\mathcal{D}}^{1}\left(\operatorname{Hom}_{S}(I, D), D\right) .
$$

The following proposition shows that in a certain sense, the conditions of Theorem 6.12 can themselves be lifted under deformation.

Proposition 6.13. Consider a flat nilpotent deformation $\mathcal{D} \longrightarrow \mathcal{C}$ and coflat $\mathcal{C}$ objects $\bar{D}$ and $\bar{E}$ with $\operatorname{Hom}_{R}(S, \bar{D})=D$ and $\operatorname{Hom}_{R}(S, \bar{E})=E$. Suppose that for certain $i \geq 0$ we have that $\operatorname{Ext}_{\mathcal{D}}^{i}\left(\operatorname{Hom}_{S}(X, D), E\right)=0$ for all $X \in \bmod (S)$. Then $\operatorname{Ext}_{\mathcal{C}}^{i}\left(\operatorname{Hom}_{R}(X, \bar{D}), \bar{E}\right)=0$ for all $X \in \bmod (R)$.

Proof. We may consider $\mathcal{C}_{S} \longrightarrow \mathcal{C}$ of order 2. By Proposition 4.7,

$$
\operatorname{Ext}_{\mathcal{C}}^{i}\left(\operatorname{Hom}_{R}(Y, \bar{D}), \bar{E}\right)=\operatorname{Ext}_{\mathcal{D}}^{i}\left(\operatorname{Hom}_{S}(Y, D), E\right)=0
$$

for all $Y \in \bmod (S)$. For $X \in \bmod (R)$, it suffices to consider $0 \longrightarrow I X \longrightarrow X \longrightarrow$ $S \otimes_{R} X \longrightarrow 0$ and the corresponding $0 \longrightarrow \operatorname{Hom}_{R}\left(S \otimes_{R} X, \bar{D}\right) \longrightarrow \operatorname{Hom}_{R}(X, \bar{D}) \longrightarrow$ $\operatorname{Hom}_{R}(I X, \bar{D}) \longrightarrow 0$. The result for $X$ then follows from the results for $Y=I X$ and $Y=S \otimes_{R} X$ via the long exact Ext-sequence.

Corollary 6.14. Consider a flat nilpotent deformation $\mathcal{D} \longrightarrow \mathcal{C}$ and a coflat $\mathcal{D}$ object D. If $\operatorname{Ext}_{\mathcal{D}}^{2}\left(\operatorname{Hom}_{S}(X, D), D\right)=0$ for all $X \in \bmod (S)$, then $\operatorname{Sk}\left(L^{c f}(D)\right) \neq \varnothing$. If $\operatorname{Ext}_{\mathcal{D}}^{2}\left(\operatorname{Hom}_{S}(X, D), D\right)=\operatorname{Ext}_{\mathcal{D}}^{1}\left(\operatorname{Hom}_{S}(X, D), D\right)=0$, then $\operatorname{Sk}\left(L^{c f}(D)\right)$ contains precisely one element. 
Proof. This follows from Propositions 4.8, 6.13 and Theorem 6.12,

Corollary 6.15. Consider a flat nilpotent deformation $\mathcal{D} \longrightarrow \mathcal{C}$ and a $\mathcal{D}$-injective $D$. Then $\operatorname{Sk}\left(L^{c f}(D)\right)$ contains precisely one lift $D \longrightarrow C$, and $C$ is a $\mathcal{C}$-injective.

Proof. We may consider $\mathcal{C}_{S} \longrightarrow \mathcal{C}$ of order 2 . The first part of the statement follows from Theorem 6.12, To prove that $C$ is injective, first note that by Proposition 4.7 $\operatorname{Ext}_{\mathcal{C}}^{i}(A, C)=0$ for all $A \in \mathcal{C}_{S}$. Finally, for $A \in \mathcal{C}$, it suffices to consider the long exact Ext sequence associated to $0 \longrightarrow I A \longrightarrow A \longrightarrow S \otimes_{R} A \longrightarrow 0$.

6.4. Deformations of categories with enough injectives. The results of the previous section allow us to prove the following theorem.

Theorem 6.16. Consider a flat nilpotent deformation $\mathcal{D} \longrightarrow \mathcal{C}$. If $\mathcal{D}$ has enough injectives, then so does $\mathcal{C}$.

Proof. We may consider $\mathcal{C}_{S} \longrightarrow \mathcal{C}$ and an object $C$ in $\mathcal{C}$. Take a $\mathcal{C}_{S}$-monomorphism $m: \operatorname{Hom}_{R}(S, C) \longrightarrow I$ to a $\mathcal{C}_{S}$-injective $I$ such that there is a lift $\bar{I}$ with $\operatorname{Hom}_{R}(S, \bar{I})$ $=I$. By Corollary 6.15, $\bar{I}$ is injective, and so $m$ can be lifted to a map $\bar{m}: C \longrightarrow \bar{I}$ with $\operatorname{Hom}_{R}(S, \bar{m})=m$. By Proposition 6.4, $\bar{m}$ is a $\mathcal{C}$-monomorphism.

This result is false for non-flat nilpotent deformations, as the following example shows.

Example 6.17. Let $k$ be a field and let $S=k, R=k[\epsilon], \epsilon^{2}=0$. Let $\mathcal{D}=\operatorname{Mod}(k)$. Let $\kappa$ be an infinite small cardinal and consider the following category:

$$
\mathcal{C}=\left\{M \in \operatorname{Mod}(R) \mid \operatorname{dim}_{k} I M \leq \kappa\right\}
$$

It is easy to see that $\mathcal{C}$ is full abelian subcategory of $\operatorname{Mod}(R)$. Clearly $\mathcal{D} \longrightarrow \mathcal{C}$ is a nilpotent deformation of order two.

A skeletal subcategory of $\operatorname{Mod}(R)$ is given by the objects

$$
V(\alpha, \beta)=k^{\oplus \alpha} \bigoplus k[\epsilon]^{\oplus \beta},
$$

where $\alpha, \beta$ are small cardinals $[23$. $\mathcal{C}$ consists of those objects $V(\alpha, \beta)$ for which $\beta \leq \kappa$.

The objects $V(\alpha, \beta)$ with $\alpha \neq 0$ are not injective in $\mathcal{C}$ since we may always replace one copy of $k$ by $k[\epsilon]$, which yields a non-split extension.

Consider an object of the form $V(\alpha, \kappa)$ in $\mathcal{C}$ with $\alpha>\kappa$ and assume that there is an injective object $E$ in $\mathcal{C}$ containing $V(\alpha, \kappa)$. Then clearly $E$ must be of the form $V\left(\alpha^{\prime}, \kappa\right)$ with $\alpha^{\prime}+\kappa \geq \alpha+\kappa$. In particular $\alpha^{\prime}>\kappa$. But then $E$ cannot be injective by the discussion in the previous paragraph. This contradiction shows that $\mathcal{C}$ does not have enough injectives.

6.5. Deformations of Grothendieck categories. The aim of this section is to prove that the property of being a Grothendieck category is preserved under flat nilpotent deformation.

For a small pre-additive category $\mathfrak{g}$ we write $\operatorname{Pr}(\mathfrak{g})=\operatorname{Mod}\left(\mathfrak{g}^{\text {op }}\right)$ for the category of (additive) presheaves on $\mathfrak{g}$. We recall the following version of the Gabriel-Popescu theorem.

Theorem $6.18(21,22,15])$. Let $\mathcal{D}$ be a Grothendieck category and let $\mathfrak{g}$ be a small generating subcategory of $\mathcal{D}$. There is a localization (i.e. a fully faithful functor with an exact left adjoint)

$$
\mathcal{D} \longrightarrow \operatorname{Pr}(\mathfrak{g}): D \longmapsto \mathcal{D}(-, D) .
$$


Conversely, if $\mathfrak{g}$ is a small pre-additive category and $\mathcal{D} \longrightarrow \operatorname{Pr}(\mathfrak{g})$ is a localization, then $\mathcal{D}$ is a Grothendieck category. Equivalence classes of such localizations are in one-to-one correspondence with so-called Gabriel (or additive) topologies on $\mathfrak{g}$.

Let $\mathcal{D}$ be a Grothendieck category with a distinguished generator $G$. We define a cardinality $|\cdot|$ on $\mathrm{Ob}(\mathcal{D})$ by

$$
|C|=|\mathcal{D}(G, C)|,
$$

where $|\mathcal{D}(G, C)|$ denotes the set theoretic cardinality of the set $\mathcal{D}(G, C)$. For a cardinal $\kappa, \mathcal{D}_{\kappa}$ is the full subcategory of $\mathcal{D}$ containing all $\mathcal{D}$-objects $D$ with $|D| \leq \kappa$.

Example 6.19. If we take $\mathcal{D}$ to be a module category $\operatorname{Mod}(A)$ over a ring $A$ and we take $G=A$, the newly defined $|M|$ coincides with the set theoretic cardinality of $M$.

The following proposition lists the essential properties of the categories $\mathcal{D}_{\kappa}$.

Proposition 6.20. If $\kappa$ is a small cardinal, then $\mathcal{D}_{\kappa}$ is essentially small. If $\kappa$ is an infinite cardinal of the form $2^{\beta}$ with $\beta \geq|G|$, then $\mathcal{D}_{\kappa}$ is a Serre subcategory of $\mathcal{D}$.

Proof. We first prove that $\mathcal{D}_{\kappa}$ is essentially small. By Theorem $6.18 \mathcal{D}_{\kappa}$ is equivalent to a full subcategory of $\operatorname{Mod}\left(\mathcal{D}(G, G)^{\mathrm{op}}\right)_{\kappa}$. The latter obviously has a small skeleton since the set of right $\mathcal{D}(G, G)$-module structures on every $\kappa^{\prime} \leq \kappa$ is small.

The fact that $\mathcal{D}_{\kappa}$ is a Serre subcategory of $\mathcal{D}$ is a consequence of Lemmas 6.21, 6.22 below, which show that the ordinary properties of cardinalities of modules more or less hold for objects in Grothendieck categories. The only not entirely obvious fact is that $\mathcal{D}_{\kappa}$ is closed under quotients under the stated hypotheses on $\kappa$. This follows from Lemma 6.22 and the following computation:

$$
(2 \kappa)^{|G|}=2^{\beta \cdot|G|}=2^{\beta}=\kappa .
$$

Lemma 6.21. For an exact sequence $0 \longrightarrow A \longrightarrow B \longrightarrow C$ in $\mathcal{D}$,

(1) $|A| \leq|B|$;

(2) $|B| \leq|A| \cdot|C|$.

Proof. This follows from the exactness of $0 \longrightarrow \mathcal{D}(G, A) \longrightarrow \mathcal{D}(G, B) \longrightarrow \mathcal{D}(G, C)$.

Lemma 6.22. For an epimorphism $f: A \longrightarrow B$,

$$
|B| \leq(2|A|)^{|G|} \text {. }
$$

Proof. For a map $g: G \longrightarrow B$, consider the pullback $P_{g}$ of $f$ and $g$ and then an epimorphism $\bigoplus_{i \in I} G \longrightarrow P_{g}$. The composition $\left(f_{i}\right)_{i \in I}: \bigoplus_{i \in I} G \longrightarrow P_{g} \longrightarrow G$ is an epimorphism. Put $J=\left\{f_{i} \mid i \in I\right\} \subset \mathcal{D}(G, G)$.

In this way we still have an epimorphism $(j)_{j \in J}: \bigoplus_{j \in J} G \longrightarrow G$ which fits in a commutative diagram

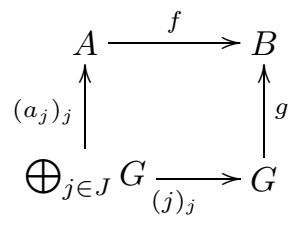


Conversely it is clear that $g$ is uniquely determined by the data $J \subset \mathcal{D}(G, G)$ and $\left(a_{j}\right)_{j \in J} \in \mathcal{D}(G, A)^{J}$, which yields the required bound.

In the remainder of this section, we consider a flat nilpotent deformation $\mathcal{D}=$ $\mathcal{C}_{S} \longrightarrow \mathcal{C}$ of order 2 in which $\mathcal{D}$ is a Grothendieck category with a fixed generator $G$ defining a cardinality $|\cdot|$ on $\mathcal{D}$. We put $\kappa=2^{\beta}$ for an infinite cardinal $\beta \geq|G|$. By Proposition 6.20, $\mathcal{D}_{\kappa}$ is an essentially small Serre subcategory of $\mathcal{D}$. We let $\mathcal{C}_{\kappa}$ be the Serre subcategory of $\mathcal{C}$ generated by $\mathcal{D}_{\kappa}$. By Lemma 6.8 $\mathcal{C}_{\kappa}$ is essentially small as well. Our aim is to show that $\mathcal{C}_{\kappa}$ generates $\mathcal{C}$.

The following lemma gives us a general procedure for constructing generators for $\mathcal{C}$. It is more general than what we need, but it will be reapplied in a slightly different setting afterwards (see Theorem 6.36).

Lemma 6.23. Let $\left(G_{i}\right)_{i \in I}$ be a (not necessarily small) collection of generators of $\mathcal{D}$. Consider a collection of objects $\left(G_{f}\right)_{f}$, indexed over all non-zero $\mathcal{C}$-maps $f$ with codomain in $\mathcal{D}$, obtained in the following manner:

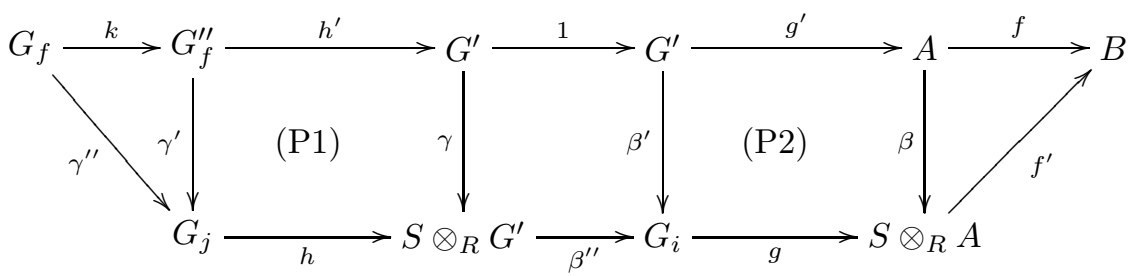

where $g$ is chosen to make $f^{\prime} \circ g \neq 0, G^{\prime}$ is the pullback of $\beta$ and $g, h$ is chosen to make $f^{\prime} \circ g \circ \beta^{\prime \prime} \circ h \neq 0, G_{f}^{\prime \prime}$ is the pullback of $\gamma$ and $h$, and $k$ is any map such that $\gamma^{\prime}=\gamma \circ k$ is an epimorphism. Then $\left(G_{f}\right)_{f}$ is a (not necessarily small) collection of generators for $\mathcal{C}$.

Proof. The $G_{f}$ clearly satisfy the generator property with respect to the maps $f: A \longrightarrow B$ with codomain in $\mathcal{D}$. We claim this is sufficient. Let $g: A \longrightarrow C$ be a general map in $\mathcal{C}$. If the composition $A \stackrel{g}{\longrightarrow} C \longrightarrow S \otimes_{R} C$ is not zero, then we are done. If the composition is zero, then we have a factorization $A \longrightarrow I C \longrightarrow C$, and we are done as well.

Lemma 6.24. Suppose $I=\{*\}, G_{*}=G$ and let $\left(G_{f}\right)_{f}$ be constructed as in Lemma 6.23 with every $k$ taken to be $1_{G_{f}^{\prime \prime}}$. Then for all $f$ we have $G_{f} \in \mathcal{C}_{\kappa}$. In particular, $\mathcal{C}$ has a small set of generators.

Proof. Recall that the squares marked with $\left(\mathrm{P}^{*}\right)$ in (6.2) are pullbacks. From (P1) it follows that we have to show that $I G^{\prime} \in \mathcal{D}_{\kappa}$. From (P2) we obtain an exact sequence

$$
0 \longrightarrow I A \longrightarrow G^{\prime} \longrightarrow G \longrightarrow 0
$$


Tensoring this sequence with $S$ we obtain a commutative diagram with exact rows and columns:

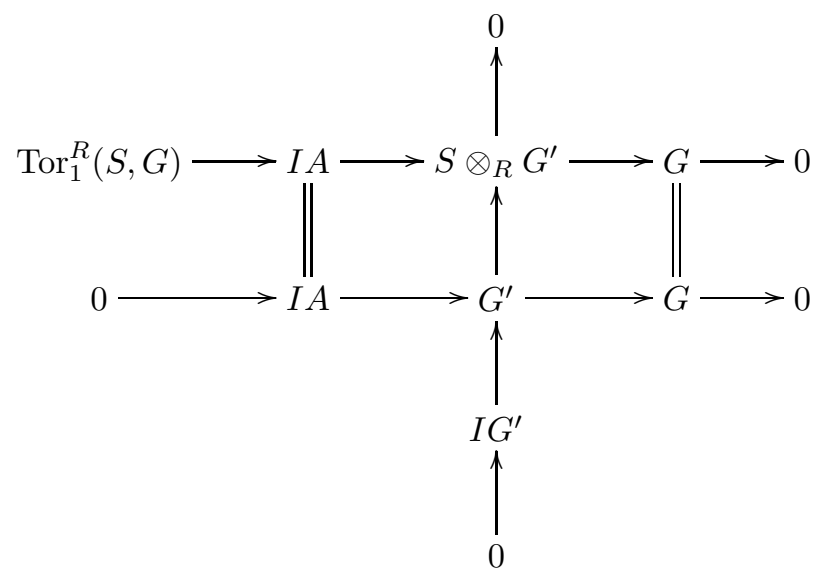

Diagram chasing yields an epimorphism $\operatorname{Tor}_{1}^{R}(S, G) \longrightarrow I G^{\prime}$, which finishes the proof.

Proposition 6.25. Let $\mathcal{E}$ be an abelian category with enough injectives. Put $\mathfrak{i}=$ $\operatorname{Inj}(\mathcal{E})$. Consider the functor

$$
\Psi: \mathcal{E}^{\mathrm{op}} \longrightarrow \operatorname{Mod}(\mathfrak{i}): C \longmapsto \mathcal{E}(C,-) .
$$

(1) $\Psi$ is exact, limit preserving and fully faithful;

(2) $\Psi$ induces an equivalence

$$
\Psi^{\prime}: \mathcal{E}^{\mathrm{op}} \longrightarrow \bmod (\mathfrak{i}) \text {. }
$$

Proof. $\Psi$ is exact and limit preserving since all the functors $\mathcal{E}(-, I): \mathcal{E}^{\mathrm{op}} \longrightarrow \mathrm{Ab}$ are exact and limit preserving.

To show that $\Psi$ is fully faithful take $C, D \in \mathrm{Ob}(\mathcal{E})$. Choose an injective copresentation

$$
0 \longrightarrow D \longrightarrow I_{0} \longrightarrow I_{1} .
$$

Then we obtain a projective presentation of $\mathcal{E}(D,-)$ in $\operatorname{Mod}(\mathfrak{i})$

$$
\mathfrak{i}\left(I_{1},-\right) \longrightarrow \mathfrak{i}\left(I_{0},-\right) \longrightarrow \mathcal{E}(D,-) \longrightarrow 0,
$$

and thus

$$
\begin{aligned}
\operatorname{Mod}(\mathfrak{i})(\mathcal{E}(D,-) & \mathcal{E}(C,-)) \\
& =\operatorname{ker}\left(\operatorname{Mod}(\mathfrak{i})\left(\mathfrak{i}\left(I_{0},-\right), \mathcal{E}(C,-)\right) \longrightarrow \operatorname{Mod}(\mathfrak{i})\left(\mathfrak{i}\left(I_{1},-\right), \mathcal{E}(C,-)\right)\right) \\
& =\operatorname{ker}\left(\mathcal{E}\left(C, I_{0}\right) \longrightarrow \mathcal{E}\left(C, I_{1}\right)\right) \\
& =\mathcal{E}(C, D) .
\end{aligned}
$$

Now consider $F \in \bmod (\mathfrak{i})$. Then $F$ has a presentation

$$
\bigoplus_{i=1}^{n} \mathfrak{i}\left(I_{i},-\right) \longrightarrow \bigoplus_{j=1}^{m} \mathfrak{i}\left(I_{j},-\right) \longrightarrow F \longrightarrow 0,
$$

hence it follows that $F$ is isomorphic to $\mathcal{E}(C,-)$ with $C$ defined by $0 \longrightarrow C \longrightarrow$ $\bigoplus_{j=1}^{m} I_{j} \longrightarrow \bigoplus_{i=1}^{n} I_{i}$. This proves that $\Psi^{\prime}$ is essentially surjective. 
Lemma 6.26. $\mathcal{C}$ has arbitrary small coproducts, and they are exact.

Proof. Besides our fixed universe $\mathcal{U}$ we introduce a larger universe $\mathcal{V}$ such that $\mathcal{C}$ is $\mathcal{V}$-small.

By Theorem 6.16, $\mathcal{C}$ has enough injectives, and putting $\mathfrak{i}=\operatorname{lnj}(\mathcal{C})$, by Proposition 6.25 we have an exact, limit preserving functor $\Psi: \mathcal{C}^{\mathrm{op}} \longrightarrow \mathcal{V}-\operatorname{Mod}(\mathfrak{i}): C \longmapsto$ $\mathcal{C}(C,-)$ inducing an equivalence $\Psi^{\prime}: \mathcal{C}^{\text {op }} \longrightarrow \mathcal{V}-\bmod (\mathfrak{i})$. We are to prove that $\mathcal{C}^{\text {op }}$ has exact $\mathcal{U}$-small products. Since $\mathcal{V}-\operatorname{Mod}(\mathfrak{i})$ has even exact $\mathcal{V}$-small products, it suffices to show that for a $\mathcal{U}$-small set of $\mathcal{C}$-objects $\left(C_{i}\right)_{i}$ the product $\prod_{i} \mathcal{C}\left(C_{i},-\right)$ in $\mathcal{V}-\operatorname{Mod}(\mathfrak{i})$ is finitely presented. Consider the short exact sequences in $\mathcal{C}$,

$$
0 \longrightarrow I C_{i} \longrightarrow C_{i} \longrightarrow S \otimes_{R} C_{i} \longrightarrow 0 .
$$

In $\mathcal{V}-\operatorname{Mod}(\mathfrak{i})$ we can take the product of the exact sequences

$$
0 \longrightarrow \mathcal{C}\left(S \otimes_{R} C_{i},-\right) \longrightarrow \mathcal{C}\left(C_{i},-\right) \longrightarrow \mathcal{C}\left(I C_{i},-\right) \longrightarrow 0
$$

to obtain an exact sequence

$$
0 \longrightarrow \mathcal{C}\left(\coprod_{i} S \otimes_{R} C_{i},-\right) \longrightarrow \prod_{i} \mathcal{C}\left(C_{i},-\right) \longrightarrow \mathcal{C}\left(\coprod_{i} I C_{i},-\right) \longrightarrow 0
$$

where we used that $\mathcal{C}_{S}$ has small $\mathcal{U}$-coproducts and $\mathcal{C}_{S} \longrightarrow \mathcal{C}$ preserves them. Then $\prod_{i} \mathcal{C}\left(C_{i},-\right)$ is finitely presented in $\mathcal{V}-\operatorname{Mod}(\mathfrak{i})$ as an extension of finitely presented objects.

The following proposition is the dual of [20, A.3.2].

Proposition 6.27. Consider an abelian category $\mathcal{E}$ with exact coproducts and any small category $\mathcal{I}$. The colimit functor

$$
\operatorname{colim}: \operatorname{Fun}(\mathcal{I}, \mathcal{E}) \longrightarrow \mathcal{E}: F \longrightarrow \operatorname{colim}(F)
$$

is trivially right exact, and its left derived functors $L_{i}$ colim exist. Moreover if $\mathcal{E}^{\prime}$ is another abelian category with exact coproducts and $\phi: \mathcal{E} \longrightarrow \mathcal{E}^{\prime}$ is an exact functor preserving coproducts, the following diagram commutes:

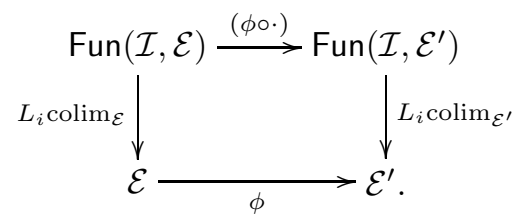

Lemma 6.28. $\mathcal{C}$ has exact filtered colimits.

Proof. By Lemma 6.26 we know that filtered colimits exist in $\mathcal{C}$, so we are to prove that for a filtered category $\mathcal{I}$, the functor

$$
\operatorname{colim}: \operatorname{Fun}(\mathcal{I}, \mathcal{C}) \longrightarrow \mathcal{C}: F \longrightarrow \operatorname{colim}(F)
$$

is exact. By Lemma 6.26 and Proposition 6.27, it suffices to prove that $L_{1}$ colim $=0$. Consider $F: \mathcal{I} \longrightarrow \mathcal{C}$. Using the exact sequences $0 \longrightarrow I F(i) \longrightarrow F(i) \longrightarrow$ $S \otimes_{R} F(i) \longrightarrow 0$ and $\phi: \mathcal{C}_{S} \longrightarrow \mathcal{C}$, we obtain an exact sequence

$$
0 \longrightarrow \phi \circ F^{\prime} \longrightarrow F \longrightarrow \phi \circ F^{\prime \prime} \longrightarrow 0
$$

for functors $F^{\prime}$ and $F^{\prime \prime}: \mathcal{I} \longrightarrow \mathcal{C}_{S}$. We obtain an exact sequence

$$
L_{1} \operatorname{colim}\left(\phi \circ F^{\prime}\right) \longrightarrow L_{1} \operatorname{colim}(F) \longrightarrow L_{1} \operatorname{colim}\left(\phi \circ F^{\prime \prime}\right),
$$


but since filtered colimits are exact in $\mathcal{C}_{S}$, it follows from Proposition 6.27 that both ends of the sequence and hence also the middle term $L_{1} \operatorname{colim}(F)$ are zero, which proves our assertion.

Theorem 6.29. Consider a flat nilpotent deformation $\mathcal{D} \longrightarrow \mathcal{C}$. If $\mathcal{D}$ is a Grothendieck category, the same holds for $\mathcal{C}$.

Proof. It suffices to consider a flat deformation $\mathcal{C}_{S} \longrightarrow \mathcal{C}$ of order 2 . The result then immediately follows from Lemmas 6.24, 6.26 and 6.28,

6.6. Deformations of locally coherent Grothendieck categories. In this section we show that local coherence of a Grothendieck category is also preserved under flat nilpotent deformation. We begin with some preliminary results.

Definition 6.30 ([13]). An object $C$ in an abelian category $\mathcal{C}$ is called fp-injective provided that a monomorphism $X \longrightarrow Y$ between finitely presented objects induces an epimorphism $\mathcal{C}(Y, C) \longrightarrow \mathcal{C}(X, C)$.

Proposition 6.31. A filtered colimit of fp-injectives is fp-injective.

Proof. Let $\operatorname{colim}_{i} C_{i}$ be a filtered colimit of fp-injectives and $0 \longrightarrow X \longrightarrow Y \longrightarrow$ $Z \longrightarrow 0$ an exact sequence of finitely presented objects. Then all sequences $0 \longrightarrow$ $\mathcal{C}\left(Z, C_{i}\right) \longrightarrow \mathcal{C}\left(Y, C_{i}\right) \longrightarrow \mathcal{C}\left(X, C_{i}\right) \longrightarrow 0$ are exact, hence so is their filtered colimit $0 \longrightarrow \mathcal{C}\left(Z, \operatorname{colim}_{i} C_{i}\right) \longrightarrow \mathcal{C}\left(Y, \operatorname{colim}_{i} C_{i}\right) \longrightarrow \mathcal{C}\left(X, \operatorname{colim}_{i} C_{i}\right) \longrightarrow 0$.

Proposition 6.32 ([13, A.1]). In a locally coherent Grothendieck category $\mathcal{C}$, the following are equivalent for $C \in \mathcal{C}$ :

(1) $C$ is fp-injective;

(2) $\operatorname{Ext}^{1}(Z, C)=0$ for every $Z$ in $\mathrm{Fp}(\mathcal{C})$;

(3) $\operatorname{Ext}^{i}(Z, C)=0$ for all $i>0$ for every $Z$ in $\operatorname{Fp}(\mathcal{C})$.

Lemma 6.33. Let $C$ be finitely presented in an abelian category $\mathcal{C}$ with enough injectives. If filtered colimits of injectives are $\mathcal{C}(C,-)$-acyclic, then $\operatorname{Ext}_{\mathcal{C}}^{n}(C,-)$ preserves filtered colimits for $n \geq 0$.

Proof. Consider a filtered colimit $\operatorname{colim}_{i} A_{i}$. By [11, we can make a functorial choice of injective resolutions $I_{i}$ of the objects $A_{i}$. Then $\operatorname{colim}_{i} I_{i}$ is a $\mathcal{C}(C,-)$ acyclic resolution of $\operatorname{colim}_{i} A_{i}$, hence we may compute

$$
\begin{aligned}
\operatorname{Ext}_{\mathcal{C}}^{n}\left(C, \operatorname{colim}_{i} A_{i}\right) & =H^{n}\left(\mathcal{C}\left(C, \operatorname{colim}_{i} I_{i}^{*}\right)\right) \\
& =H^{n}\left(\operatorname{colim}_{i} \mathcal{C}\left(C, I_{i}^{*}\right)\right) \\
& =\operatorname{colim}_{i} \operatorname{Ext}_{\mathcal{C}}^{n}\left(C, A_{i}\right) .
\end{aligned}
$$

Proposition 6.34. Let $C$ be finitely presented in a locally coherent Grothendieck category $\mathcal{C}$. Then $\operatorname{Ext}_{\mathcal{C}}^{n}(C,-)$ preserves filtered colimits for $n \geq 0$.

Proof. This follows from Proposition 6.32 and Lemma 6.33.

Lemma 6.35. Consider a flat nilpotent deformation of Grothendieck categories $\mathcal{C}_{S} \longrightarrow \mathcal{C}$ with $\mathcal{C}_{S}$ locally coherent. For a finitely presented object $C$ of $\mathcal{C}_{S}, \operatorname{Ext}_{\mathcal{C}}^{n}(C,-)$ preserves filtered colimits for $n \geq 0$. 
Proof. By Lemma 6.33 it suffices to show that for a filtered colimit colim $I_{i}$ of injectives, $\operatorname{Ext}_{\mathcal{C}}^{n}\left(C, \operatorname{colim}_{i} I_{i}\right)=0$. Now by Corollary 2.8, $\operatorname{colim}_{i} I_{i}$ is coflat. Consequently, we may use Propositions 4.7 and 6.34 to compute that

$$
\begin{aligned}
\operatorname{Ext}_{\mathcal{C}}^{n}\left(C, \operatorname{colim}_{i} I_{i}\right) & =\operatorname{Ext}_{\mathcal{C}_{S}}^{n}\left(C, \operatorname{Hom}_{R}\left(S, \operatorname{colim}_{i} I_{i}\right)\right) \\
& =\operatorname{Ext}_{\mathcal{C}_{S}}^{n}\left(C, \operatorname{colim}_{i} \operatorname{Hom}_{R}\left(S, I_{i}\right)\right) \\
& =\operatorname{colim}_{i} \operatorname{Ext}_{\mathcal{C}_{S}}^{n}\left(C, \operatorname{Hom}_{R}\left(S, I_{i}\right)\right) \\
& =\operatorname{colim}_{i} \operatorname{Ext}_{\mathcal{C}}^{n}\left(C, I_{i}\right)=0 . \quad \square
\end{aligned}
$$

We are now able to prove the main theorem.

Theorem 6.36. Consider a flat nilpotent deformation $\mathcal{D} \longrightarrow \mathcal{C}$ of Grothendieck categories. If $\mathcal{D}$ is locally coherent, the same holds for $\mathcal{C}$.

Proof. We may consider $\mathcal{C}_{S} \longrightarrow \mathcal{C}$ of order 2 . Let $\left(G_{i}\right)_{i}$ be a small set of coherent generators of $\mathcal{C}_{S}$. We want to carry out the construction of Lemma 6.23 for specific maps $k$. Suppose we have constructed the objects $G_{f}^{\prime \prime}$. Since $\mathcal{C}_{S}$ is locally coherent, $I G^{\prime}$ is a small filtered colimit $I G^{\prime}=\operatorname{colim}_{j} F_{j}$ of coherent objects $F_{j}$ of $\mathcal{C}_{S}$. Every canonical map $F_{i} \longrightarrow I G^{\prime}$ induces a map $\operatorname{Ext}_{\mathcal{C}}^{1}\left(G_{j}, F_{i}\right) \longrightarrow \operatorname{Ext}_{\mathcal{C}}^{1}\left(G_{j}, I G^{\prime}\right)$ mapping an extension $0 \longrightarrow F_{i} \longrightarrow E \longrightarrow G_{j} \longrightarrow 0$ to the pullback

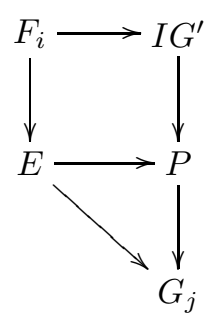

By Lemma 6.35, $\operatorname{Ext}_{\mathcal{C}}^{1}\left(G_{j},-\right)$ preserves filtered colimits, hence

$$
\operatorname{Ext}_{\mathcal{C}}^{1}\left(G_{j}, I G^{\prime}\right)=\operatorname{colim}_{i} \operatorname{Ext}_{\mathcal{C}}^{1}\left(G_{j}, F_{i}\right) .
$$

It then follows from the construction of this filtered colimit that the extension

$$
0 \longrightarrow I G^{\prime} \underset{\delta^{\prime}}{\longrightarrow} G_{f}^{\prime \prime} \underset{\gamma^{\prime}}{\longrightarrow} G_{j} \longrightarrow 0
$$

is in the image of one of the maps $\operatorname{Ext}_{\mathcal{C}}^{1}\left(G_{j}, F_{i}\right) \longrightarrow \operatorname{Ext}_{\mathcal{C}}^{1}\left(G_{j}, I G^{\prime}\right)$. Hence we obtain an extension

$$
0 \longrightarrow F_{i} \underset{\delta^{\prime \prime}}{\longrightarrow} G_{f} \underset{\gamma^{\prime \prime}}{\longrightarrow} G_{j} \longrightarrow 0
$$

and a map $k: G_{f} \longrightarrow G_{f}^{\prime \prime}$ as in Lemma 6.23. Hence $\left(G_{f}\right)_{f}$ is a set of generators for $\mathcal{C}$. Now (see 2.2$) \operatorname{Fp}\left(\mathcal{C}_{S}\right)$ has a small skeleton, and since $\mathcal{C}$ has small Yoneda Ext groups, it follows that the image of $\left(G_{f}\right)_{f}$ is small. Using Proposition 4.6, it is readily seen that the objects $G_{i}$ and $F_{i}$ are coherent in $\mathcal{C}$. It then follows that all objects of $\left(G_{f}\right)_{f}$ are finitely generated, hence $\mathcal{C}$ is locally finitely generated. But then all objects of $\left(G_{f}\right)_{f}$ are finitely presented, hence $\mathcal{C}$ is locally finitely presented. Finaly all objects of $\left(G_{f}\right)_{f}$ are coherent, hence it follows that $\mathcal{C}$ is locally coherent, as we set out to prove. 


\section{DEFORMATION AND LOCALIZATION}

7.1. Statement of the main results. A fully faithful functor $i: \mathcal{L} \longrightarrow \mathcal{C}$ between Grothendieck categories is called a localization if it has an exact left adjoint. If in addition $i$ is an embedding of a full subcategory closed under isomorphisms, then we call $i$ a strict localization. In this section, we study the compatibility of localization with deformations.

Below we prove the following result.

Theorem 7.1. Let $\mathcal{C}_{S} \longrightarrow \mathcal{C}$ be a nilpotent deformation of Grothendieck categories. Then

$$
\mathcal{L} \longmapsto \mathcal{L}_{S}
$$

induces a bijection between the sets of strict localizations of $\mathcal{C}$ and $\mathcal{C}_{S}$.

Note that this theorem does not say that any nilpotent deformation of a localization of $\mathcal{C}_{S}$ is itself a localization of $\mathcal{C}$. This is in fact false (even under the appropriate flatness hypotheses).

Example 7.2. Consider a field $S=k . R=k[\epsilon], \epsilon^{2}=0 . \Lambda=k[x, y] . \mathcal{C}=\operatorname{Mod}(\Lambda)$, $\mathcal{L}=\operatorname{Mod}\left(\Lambda_{x}\right)$. By Theorem 8.16 below the deformations of $\mathcal{C}$ and $\mathcal{L}$ correspond to the deformations of $\Lambda$ and $\Lambda_{x}$. These are respectively given by the rings

$$
\begin{gathered}
R[x, y] /(y x-x y-f \epsilon) \quad \text { and } \\
R\left[x, x^{-1}, y\right] /(y x-x y-g \epsilon)
\end{gathered}
$$

for $f \in k[x, y], g \in k\left[x, x^{-1}, y\right]$. Clearly $\Lambda_{x}$ has many more deformations than $\Lambda$.

The next theorem allows us to recognize those deformations of localizations which are themselves localizations.

Theorem 7.3. Consider a commutative diagram

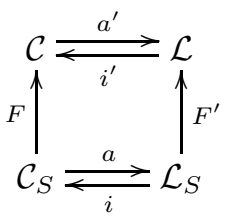

in which $F$ and $F^{\prime}$ are flat nilpotent deformations of Grothendieck categories and $(a, i)$ and $\left(a^{\prime}, i^{\prime}\right)$ are pairs of adjoint functors. Suppose $i^{\prime}$ maps injectives onto coflats. If $i$ is a localization, then the same holds for $i^{\prime}$.

If $u: \mathfrak{u} \longrightarrow \mathcal{C}$ is an additive functor from a small pre-additive category $\mathfrak{u}$ to a Grothendieck category $\mathcal{C}$, then we have a pair of adjoint functors $(a, i)$, where $i: \mathcal{C} \longrightarrow \operatorname{Pr}(\mathfrak{u}): U \longmapsto \mathcal{C}(u(-), U)$ and $a: \operatorname{Pr}(\mathfrak{u}) \longrightarrow \mathcal{C}$ is the unique colimit preserving functor sending $\mathfrak{u}(-, A)$ to $u(A)$. We say that $u$ induces a localization if $i$ is a localization.

We will apply Theorem 7.3 in the following setting: assume that $R \longrightarrow S$ has nilpotent kernel and consider a commutative diagram

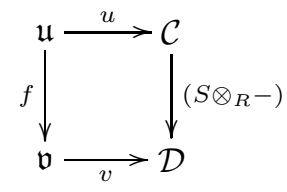


where the categories and functors are as follows:

(1) $\mathfrak{u}, \mathfrak{v}$ are, respectively, small $R$ and $S$-linear flat categories;

(2) the functor $f$ is a nilpotent linear deformation;

(3) $\mathcal{C}, \mathcal{D}$ are, respectively, $R$ and $S$-linear flat Grothendieck categories;

(4) $u, v$ are, respectively, $R$ and $S$-linear additive functors;

(5) the functor $S \otimes_{R}$ - is the left adjoint to a deformation $\mathcal{D} \longrightarrow \mathcal{C}$;

(6) the images of $u, v$ consist of flat objects.

Proposition 7.4. If $v$ induces a localization $\mathcal{D} \longrightarrow \operatorname{Pr}(\mathfrak{v})$, then $u$ induces a localization $\mathcal{C} \longrightarrow \operatorname{Pr}(\mathfrak{u})$.

7.2. Proofs. Let $\mathcal{C}$ be an abelian category. Recall that a full subcategory $\mathcal{S} \subset \mathcal{C}$ closed under subquotients and extensions in called a Serre subcategory of $\mathcal{C}$. If moreover $\mathcal{C}$ is Grothendieck and $\mathcal{S}$ is closed under coproducts, then $\mathcal{S}$ is called a localizing subcategory of $\mathcal{C}$. If $\mathcal{S}$ is localizing, then the quotient category $\mathcal{C} / \mathcal{S}$ is again a Grothendieck category and the composition $\mathcal{S}^{\perp} \longrightarrow \mathcal{C} \longrightarrow \mathcal{C} / \mathcal{S}$, where $\mathcal{S}^{\perp}$ is the full subcategory of $\mathcal{S}$ with objects

$$
\operatorname{Ob}\left(\mathcal{S}^{\perp}\right)=\left\{C \in \mathcal{C} \mid \forall S \in \mathcal{S}: \operatorname{Ext}_{\mathcal{C}}^{i}(S, C)=0, i=0,1\right\},
$$

is an equivalence of categories.

Assume that $i: \mathcal{L} \longrightarrow \mathcal{C}$ is a localization of Grothendieck categories with exact left adjoint $a$. Then $\operatorname{ker}(a)$ is a localizing subcategory and $a$ induces an equivalence $\mathcal{C} / \operatorname{ker}(a) \longrightarrow \mathcal{L}$.

If $i$ is a strict localization, then $\mathcal{L}=\operatorname{ker}(a)^{\perp}$. We summarize this discussion in the following proposition.

Proposition 7.5. Consider a Grothendieck category $\mathcal{C}$. Let $\mathfrak{s}$ denote the set of localizing subcategories of $\mathcal{C}$ and let $\mathfrak{l}$ denote the set of strict localizations of $\mathcal{C}$. Then

$$
\mathfrak{s} \longrightarrow \mathfrak{l}: \mathcal{S} \longmapsto \mathcal{S}^{\perp}
$$

and

$$
\mathfrak{l} \longrightarrow \mathfrak{s}:(\mathcal{L} \stackrel{i}{\rightarrow} \mathcal{C}) \longmapsto \operatorname{ker}(a),
$$

where $a$ is a left adjoint of $i$, are inverse bijections.

For a set of objects or a subcategory $\mathcal{S} \subset \mathcal{C}$ we will write $\langle\mathcal{S}\rangle_{\mathcal{C}}$ for the smallest Serre subcategory of $\mathcal{C}$ containing $\mathcal{S}$.

Now assume that $R, S, I$ have their usual meaning and assume $I^{n}=0$. Let $\mathcal{C}$ be an $R$-linear abelian category.

Proposition 7.6. Let $\mathfrak{s}^{\prime}$ denote the set of Serre subcategories of $\mathcal{C}$ and $\mathfrak{s}$ the set of Serre subcategories of $\mathcal{C}_{S}$. Then

$$
\mathfrak{s}^{\prime} \longrightarrow \mathfrak{s}: \mathcal{S}^{\prime} \longmapsto \mathcal{S}^{\prime} \cap \mathcal{C}_{S}
$$

and

$$
\mathfrak{s} \longrightarrow \mathfrak{s}^{\prime}: \mathcal{S} \longmapsto\langle\mathcal{S}\rangle_{\mathcal{C}}
$$

are inverse bijections. If $\mathcal{C}$ is a Grothendieck category, they restrict to inverse bijections between the respective sets of localizing subcategories.

Proof. Let $\mathcal{S}, \mathcal{S}^{\prime}$ be objects in $\mathfrak{s}$ and $\mathfrak{s}^{\prime}$, respectively. Any object $C$ in $\mathcal{C}$ has a finite filtration

$$
0=I^{n} C \subseteq I^{n-1} C \subseteq \cdots \subseteq I^{2} C \subseteq I C \subseteq I^{0} C=C .
$$


Write $\operatorname{gr} C \in \mathcal{C}_{S}$ for its associated graded object. The formation of $\operatorname{gr} C$ is compatible with coproducts.

For $\mathcal{S} \in \mathfrak{s}$ let $\overline{\mathcal{S}}$ be the full subcategory of $\mathcal{C}$ whose objects are given by

$$
\operatorname{Ob}(\overline{\mathcal{S}})=\{C \in \mathcal{C} \mid \operatorname{gr} C \in \mathcal{S}\} .
$$

It is easy to see that $\overline{\mathcal{S}}$ is a Serre subcategory of $\mathcal{C}$ which is localizing if $\mathcal{S}$ is localizing.

Clearly $\mathcal{S} \subset \overline{\mathcal{S}} \subset\langle\mathcal{S}\rangle_{\mathcal{C}}$, and since $\overline{\mathcal{S}}$ is Serre, we deduce $\langle\mathcal{S}\rangle_{\mathcal{C}}=\overline{\mathcal{S}}$. This immediately implies

$$
\langle\mathcal{S}\rangle_{\mathcal{C}} \cap \mathcal{C}_{S}=\mathcal{S}
$$

Clearly if $\mathcal{S}^{\prime} \in \mathfrak{s}^{\prime}$, then $\mathcal{S}^{\prime} \cap \mathcal{C}_{S} \in \mathfrak{s}$, and since colimits in $\mathcal{C}$ and $\mathcal{C}_{S}$ are computed in the same way (Proposition 4.2), $\mathcal{S}^{\prime} \cap \mathcal{C}_{S}$ is localizing if $\mathcal{S}^{\prime}$ is localizing. We certainly have

$$
\left\langle\mathcal{S}^{\prime} \cap \mathcal{C}_{S}\right\rangle_{\mathcal{C}} \subset \mathcal{S}^{\prime}
$$

However if $C \in \mathcal{S}^{\prime}$, then $\operatorname{gr} C \in \mathcal{S}^{\prime} \cap \mathcal{C}_{S}$ and hence $C \in\left\langle\mathcal{S}^{\prime} \cap \mathcal{C}_{S}\right\rangle_{\mathcal{C}}$ by the earlier discussion. Thus in fact $\left\langle\mathcal{S}^{\prime} \cap \mathcal{C}_{S}\right\rangle_{\mathcal{C}}=\mathcal{S}^{\prime}$, and we are done.

Proof of Theorem 7.1. Let $i: \mathcal{L} \longrightarrow \mathcal{C}$ be a strict localization and let $a: \mathcal{C} \longrightarrow \mathcal{L}$ be the exact left adjoint to $i$. Consider the pair of functors $\left(i_{S}, a_{S}\right)$ between $\mathcal{L}_{S}$ and $\mathcal{C}_{S}$. It is clear that $i_{S}$ is still fully faithful and that $a_{S}$ is an exact left adjoint to $i_{S}$. Thus $i_{S}: \mathcal{L}_{S} \longrightarrow \mathcal{C}_{S}$ is a (strict) localization.

Let $\mathfrak{l}^{\prime}$ denote the set of strict localizations of $\mathcal{C}$ and $\mathfrak{l}$ the set of strict localizations of $\mathcal{C}_{S}$. Propositions 7.5 and 7.6 furnish us with bijections

$$
\mathfrak{l}^{\prime} \longrightarrow \mathfrak{s}^{\prime} \longrightarrow \mathfrak{s} \longrightarrow \mathfrak{l}
$$

whose composition sends $\mathcal{L}$ to $\left(\operatorname{ker}(a) \cap \mathcal{C}_{S}\right)^{\perp}=\operatorname{ker}\left(a_{S}\right)^{\perp}=\mathcal{L}_{S}$, finishing the proof.

The proof of Theorem $[7.3$ is based on the following observation.

Proposition 7.7. Consider a functor $i: \mathcal{L} \longrightarrow \mathcal{C}$ between Grothendieck categories and suppose $i$ has a left adjoint $a$. The following are equivalent:

(1) $i$ is a localization;

(2) $i$ preserves injectives and $i: \operatorname{lnj}(\mathcal{L}) \longrightarrow \operatorname{Inj}(\mathcal{C})$ is fully faithful.

Proof. Obviously (1) implies (2). Suppose (2) holds. It is easily seen that $i$ is fully faithful. The exactness of $a$ follows since exactness of a sequence can be tested by considering Hom's into all injectives.

Proof of Theorem 7.3. We use Proposition 7.7. First, let us show that $i^{\prime}$ preserves injectives. Consider $E \in \operatorname{lnj}(\mathcal{L})$. To prove that $i^{\prime}(E)$ is injective in $\mathcal{C}$, it suffices that for all $C \in \mathcal{C}_{S}$ we have $\operatorname{Ext}_{\mathcal{C}}^{1}\left(C, i^{\prime}(E)\right)=\operatorname{Ext}_{\mathcal{C}_{S}}^{1}\left(C, \operatorname{Hom}_{R}\left(S, i^{\prime}(E)\right)\right)=0$, where the first equality follows from Proposition 4.7.

Looking at right adjoints in (7.1) we obtain a commutative diagram

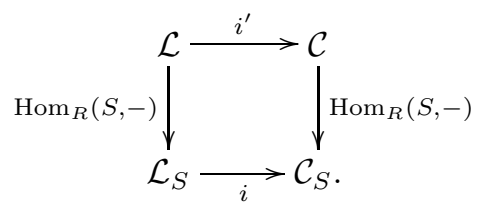

The desired result follows from the fact that $\operatorname{Hom}_{R}\left(S, i^{\prime}(E)\right)=i\left(\operatorname{Hom}_{R}(S, E)\right)$ is injective in $\mathcal{C}_{S}$. Next, we are to prove that $\eta: \mathcal{L}\left(I, I^{\prime}\right) \longrightarrow \mathcal{C}\left(i^{\prime}(I), i^{\prime}\left(I^{\prime}\right)\right)$ is an 
isomorphism for injectives $I$ and $I^{\prime}$. Since $\mathcal{L}\left(I, I^{\prime}\right)$ and $\mathcal{C}\left(i^{\prime}(I), i^{\prime}\left(I^{\prime}\right)\right)$ are flat in $\operatorname{Mod}(R)$, using the 5 -lemma it suffices that $S \otimes_{R} \eta$ is an isomorphism. But $S \otimes_{R} \eta$ is isomorphic to

$$
\mathcal{L}_{S}\left(\operatorname{Hom}_{R}(S, I), \operatorname{Hom}_{R}\left(S, I^{\prime}\right)\right) \longrightarrow \mathcal{C}_{S}\left(i\left(\operatorname{Hom}_{R}(S, I)\right), i\left(\operatorname{Hom}_{R}\left(S, I^{\prime}\right)\right)\right),
$$

which proves our assertion.

Proof of Proposition 7.4. We apply Theorem 7.3. We have a diagram

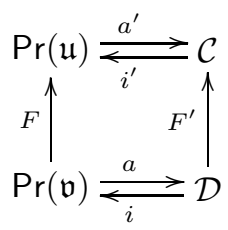

where $(a, i),\left(a^{\prime}, i^{\prime}\right)$ are the pairs of adjoint functors associated to $v$ and $u$; $F^{\prime}$ is the deformation we started with and $F$ is the abelian deformation associated to the linear deformation $f^{\mathrm{op}}: \mathfrak{u}^{\mathrm{op}} \longrightarrow \mathfrak{v}^{\mathrm{op}}$ (see Proposition 5.4).

We claim that (7.3) is commutative. We first consider the " $i$-square". Starting with $D \in \mathcal{D}$ we have

$$
\begin{aligned}
(F \circ i)(D) & =\mathcal{D}((v \circ f)(-), D) \\
& =\mathcal{D}\left(S \otimes_{R} u(-), D\right) \\
& =\mathcal{C}\left(u(-), F^{\prime}(D)\right) \\
& =\left(i^{\prime} \circ F^{\prime}\right)(D) .
\end{aligned}
$$

Since $F, F^{\prime}$ are fully faithful any $R$-linear functor $G$ between $\operatorname{Pr}(\mathfrak{u})$ and $\mathcal{C}$ (in both directions) restricts to exactly one functor between $\operatorname{Pr}(\mathfrak{v})$ and $\mathcal{D}$ (up to natural isomorphism) necessarily given by $G_{S}$. A pair of adjoint functors $(G, H)$ restricts to a pair of adjoint functors $\left(G_{S}, H_{S}\right)$.

Thus we obtain a pair of adjoint functors $\left(a_{S}^{\prime}, i_{S}^{\prime}\right)$ between $\operatorname{Pr}(\mathfrak{u})$ and $\mathcal{C}$, and furthermore $i=i_{S}^{\prime}$. Since $a$ is the left adjoint to $i$ we obtain $a=a_{S}^{\prime}$ (up to natural isomorphism). This implies the commutivity of the " $a$-square" in (7.3).

The remaining hypothesis of Theorem 7.3 that we need to check is that $i^{\prime}$ sends injectives to coflats. If $E \in \mathcal{C}$ is injective, then it is coflat by Proposition 3.4. Since the objects of $\mathfrak{u}$ are mapped to flat objects by $u$, this implies that $\mathcal{C}(u(-), E)$ takes on coflat values by Proposition 2.9(7). Hence by Proposition 2.10 $i^{\prime}(E)$ is coflat.

\section{Equivalent DEFormation PROBLems}

In this section we obtain several "equivalences of deformation problems". We start by formalizing what we mean by this.

8.1. Deformation pseudofunctors. In this section we need to be careful about our choices of universe. Therefore we make them temporarily explicit in our notations.

Let $\mathcal{U}$ be a universe. We will denote by $\mathcal{U}-\mathrm{Rng}^{0}$ the category with coherent commutative $\mathcal{U}$-rings as objects and surjective ring maps with a finitely generated, nilpotent kernel as morphisms. For a fixed coherent ring $S \in \mathcal{U}$, we consider the category $\mathcal{U}-\mathrm{Rng}^{0} / S$. 
Fix some other universe $\mathcal{W}$. A deformation pseudofunctor is by definition a pseudofunctor

$$
D: \mathcal{U}-\mathrm{Rng}^{0} / S \longrightarrow \mathcal{W}-\mathrm{Gd}
$$

An equivalence of deformation pseudofunctors $D_{1}, D_{2}: \mathcal{U}-\operatorname{Rng}^{0} / S \longrightarrow \mathcal{W}-\mathrm{Gd}$ is a pseudonatural transformation $\mu: D_{1} \longrightarrow D_{2}$ for which every $D_{1}(R) \longrightarrow D_{2}(R)$ is an equivalence of categories. It is easy to see that this defines an equivalence relation on deformation pseudofunctors.

The dependence of our notations on the universes $\mathcal{U}$ and $\mathcal{W}$ is a nuisance, but the deformation pseudofunctors we will consider below will be stable under enlarging $\mathcal{U}$ and $\mathcal{W}$ in a suitable sense. The following proposition is a first step in this direction.

Proposition 8.1. Let $\mathcal{U} \subset \mathcal{U}^{\prime}$ be universes. Then the obvious functor

$$
\mathcal{U}-\mathrm{Rng}^{0} / S \longrightarrow \mathcal{U}^{\prime}-\mathrm{Rng}^{0} / S
$$

is an equivalence of categories. Consequently, two deformation pseudofunctors $D_{1}, D_{2}: \mathcal{U}^{\prime}-\mathrm{Rng}^{0} / S \longrightarrow \mathcal{W}-\mathrm{Gd}$ are equivalent if and only if their restrictions to $\mathcal{U}-\mathrm{Rng}^{0} / S$ are equivalent.

Proof. We only need to show that it is essentially surjective, and this readily follows from Lemma 6.5

\subsection{Abelian and linear deformations.}

Definition 8.2. (1) Consider an $S$-linear category $\mathfrak{b}$. An equivalence of deformations from $f_{1}: \mathfrak{a}_{1} \longrightarrow \mathfrak{b}$ to $f_{2}: \mathfrak{a}_{2} \longrightarrow \mathfrak{b}$ is an equivalence of $R$-linear categories $\varphi: \mathfrak{a}_{2} \longrightarrow \mathfrak{a}_{1}$ such that $f_{1} \circ \varphi$ is naturally isomorphic to $f_{2}$. If $\varphi$ is an isomorphism with $f_{1} \circ \varphi=f_{2}$, it is called an isomorphism of deformations.

(2) Consider an abelian $S$-linear category $\mathcal{D}$. An equivalence of deformations from $F_{1}: \mathcal{D} \longrightarrow \mathcal{C}_{1}$ to $F_{2}: \mathcal{D} \longrightarrow \mathcal{C}_{2}$ is an equivalence of $R$-linear categories $\Phi: \mathcal{C}_{1} \longrightarrow \mathcal{C}_{2}$ such that $\Phi \circ F_{1}$ is naturally isomorphic to $F_{2}$. If $\Phi$ is an isomorphism with $\Phi \circ F_{1}=F_{2}$, it is called an isomorphism of deformations.

We consider the following groupoids for $R \in \mathcal{U}-\mathrm{Rng}^{0} / S$ (where $S \in \mathcal{U}$ ):

(1) For a flat $S$-linear $\mathcal{U}$-category $\mathfrak{b}$ and a universe $\mathcal{V}$ such that $\mathfrak{b}$ is essentially $\mathcal{V}$-small and $\mathcal{U} \in \mathcal{V}$, we consider the groupoid $\mathcal{V}$ - $\operatorname{def}_{\mathfrak{b}}(R)$. The objects of $\mathcal{V}$ - $\operatorname{def}_{\mathfrak{b}}(R)$ are flat $R$-deformations of $\mathfrak{b}$ which are elements of $\mathcal{V}$. Its morphisms are equivalences of deformations modulo natural isomorphism of functors.

(2) For a flat $S$-linear abelian $\mathcal{U}$-category $\mathcal{D}$ and a universe $\mathcal{V}$ such that $\mathcal{D}$ is essentially $\mathcal{V}$-small and $\mathcal{U} \in \mathcal{V}$, we consider the groupoid $\mathcal{V}$ - $\operatorname{Def}_{\mathcal{D}}(R)$. The objects of $\mathcal{V}-\operatorname{Def}_{\mathcal{D}}(R)$ are the flat $R$-deformations of $\mathcal{D}$ which are elements of $\mathcal{V}$. Its morphisms are equivalences of deformations modulo natural isomorphism of functors.

Clearly $\operatorname{Ob}\left(\mathcal{V}-\operatorname{def}_{\mathfrak{b}}(R)\right) \subset \mathcal{V}$ and $\operatorname{Ob}\left(\mathcal{V}-\operatorname{Def}_{\mathcal{D}}(R)\right) \subset \mathcal{V}$. Hence if we take a universe $\mathcal{W}$ with $\mathcal{V} \in \mathcal{W}$, this yields deformation pseudofunctors

$$
\mathcal{V}-\operatorname{def}_{\mathfrak{b}}, \mathcal{V}-\operatorname{Def}_{\mathcal{D}}: \mathcal{U}-\mathrm{Rng}^{0} / S \longrightarrow \mathcal{W}-\mathrm{Gd}
$$

The universe $\mathcal{W}$ is a purely technical device to make sure $\mathcal{V}-\operatorname{def}_{\mathfrak{b}}, \mathcal{V}-\operatorname{Def}_{\mathcal{D}}$ take their values in a category. Obviously, the equivalence or non-equivalence of 
two deformation pseudofunctors $D_{1}, D_{2}: \mathcal{U}-\mathrm{Rng}^{0} / S \longrightarrow \mathcal{W}-\mathrm{Gd}$ is preserved under changing from $\mathcal{W}$ to $\mathcal{W}^{\prime}$ for some $\mathcal{W} \subset \mathcal{W}^{\prime}$.

The following proposition shows that the choice of the universe $\mathcal{V}$ is harmless as well. Consider universes $\mathcal{V} \subset \mathcal{V}^{\prime}$ with $\mathcal{V}^{\prime} \in \mathcal{W}$.

Proposition 8.3. Let $\mathfrak{b}$ be a flat $S$-linear $\mathcal{U}$-category and let $\mathcal{D}$ be a flat $S$-linear abelian $\mathcal{U}$-category. Then the obvious pseudonatural tranformations

$$
\begin{aligned}
\mathcal{V}-\operatorname{def}_{\mathfrak{b}} & \longrightarrow \mathcal{V}^{\prime}-\operatorname{def}_{\mathfrak{b}}, \\
\mathcal{V}-\operatorname{Def}_{\mathcal{D}} & \longrightarrow \mathcal{V}^{\prime}-\operatorname{Def}_{\mathcal{D}}
\end{aligned}
$$

are equivalences of deformation pseudofunctors.

Proof. This follows from Propositions 6.7 6.9.

8.3. Small skeletons. From now on, we will simply write $\operatorname{def}_{\mathfrak{b}}$ and $\operatorname{Def}_{\mathcal{D}}$ to denote the functors $\mathcal{V}-\operatorname{def}_{\mathfrak{b}}$ and $\mathcal{V}-\operatorname{Def}_{\mathcal{D}}$ for some $\mathcal{U}, \mathcal{V}$ and $\mathcal{W}$ as above. Below we fix the universe $\mathcal{U}$. We assume that all rings are in $\mathcal{U}$ and write $\operatorname{Rng}^{0}$ for $\mathcal{U}-\mathrm{Rng}^{0}$. The notions of small and essentially small are as usual with respect to the fixed universe $\mathcal{U}$. The same holds for the notion of a Grothendieck category.

We prove the following results.

Theorem 8.4. Assume that $\mathfrak{b}$ is an essentially small flat $S$-linear category. Then $\operatorname{def}_{\mathfrak{b}}(R)$ has a small skeleton.

Proof. Since $\mathfrak{b}$ is assentially small, there is an infinite small cardinal $\kappa$ with $|\operatorname{Sk}(\mathfrak{b})| \leq$ $\kappa$ and $\left|\mathfrak{b}\left(B, B^{\prime}\right)\right| \leq \kappa$ for all $B, B^{\prime} \in \mathfrak{b}$. By Lemma 6.6, the same bound holds for every $\mathfrak{a} \in \operatorname{def}_{\mathfrak{b}}(R)$. It is easily seen that up to equivalence, the number of $R$-linear categories $\mathfrak{a}$ satisfying this bound is small. Furthermore, for a given $\mathfrak{a}$, up to natural isomorphism, the number of equivalences $S \otimes_{R} \mathfrak{a} \longrightarrow \mathfrak{b}$ is small (see Lemma 2.3).

Theorem 8.5. Assume that $\mathcal{D}$ is a flat $S$-linear abelian category which is either essentially small or a Grothendieck category. Then $\operatorname{Def}_{\mathcal{D}}(R)$ has a small skeleton.

Proof. We may assume that $I^{2}=0$. Assume first that $\mathcal{D}$ is essentially small. Then there is an infinite small cardinal $\kappa$ with $|\operatorname{Sk}(\mathcal{D})| \leq \kappa$ and $\left|\mathcal{D}\left(D, D^{\prime}\right)\right| \leq \kappa$ for all $D, D^{\prime} \in \mathcal{D}$. By Lemma 6.8, the same bound holds for every $\mathcal{C} \in \operatorname{Def}_{\mathcal{D}}(R)$. The proof is then finished as in the proof of Theorem 8.4.

Now assume that $\mathcal{D}$ is a Grothendieck category with a generator $G$. Let $\kappa$ and $\mathcal{D}_{\kappa}$ be as in 6.5 . If $\mathcal{D} \longrightarrow \mathcal{C}$ is a deformation, the image $G^{\prime}$ of $G$ in $\mathcal{C}_{S}$ defines a cardinality on $\mathcal{C}_{S}$ which allows us to define $\mathcal{C}_{\kappa}$ as in 6.5. By Proposition 6.20, we can take an infinite small cardinal $\lambda$ with $\left|\operatorname{Sk}\left(\mathcal{D}_{\kappa}\right)\right| \leq \lambda$ and $\left|\mathcal{D}\left(D, D^{\prime}\right)\right| \leq \lambda$ for all $D, D^{\prime} \in \mathcal{D}_{\kappa}$. By Lemma 6.8, the same bound holds for $\mathcal{C}_{\kappa}$ for every $\mathcal{C} \in \operatorname{Def}_{\mathcal{D}}(R)$, and by Lemmas 6.23, 6.24, $\mathcal{C}_{\kappa}$ generates $\mathcal{C}$. Consequently, using Theorem 6.18, up to equivalence, a deformation $\mathcal{D} \longrightarrow \mathcal{C}$ is determined by:

(1) an $R$-linear category $\mathfrak{g}$ with $|\mathrm{Ob}(\mathfrak{g})| \leq \lambda$ and $\left|\mathfrak{g}\left(G, G^{\prime}\right)\right| \leq \lambda$ for all $G, G^{\prime} \in \mathfrak{g}$;

(2) an additive topology on $\mathfrak{g}$;

(3) an object $G^{\prime} \in \mathfrak{g}$ and a map $\mathcal{D}(G, G) \longrightarrow \mathfrak{g}\left(G^{\prime}, G^{\prime}\right)$.

Clearly, the number of such data is small, which finishes the proof.

Remark 8.6. Example6.17 shows that Theorem 8.5 is trivially false without the flatness requirement on deformations, even for $\mathcal{D}=\operatorname{Mod}(k), k$ a field and $R=k[\epsilon] /\left(\epsilon^{2}\right)$. 
Indeed every small cardinal $\kappa$ in Example 6.17 yields a different deformation of $\mathcal{D}$ and the set of small cardinals is not itself small.

8.4. Elementary deformation equivalences. We have the following elementary deformation equivalences.

Proposition 8.7. (1) For every flat $S$-linear $\mathfrak{b}$ there is an equivalence

$$
\theta: \operatorname{def}_{\mathfrak{b}} \longrightarrow \operatorname{def}_{\mathfrak{b}^{\text {op }}}
$$

(2) an equivalence $F: \mathfrak{b} \longrightarrow \mathfrak{b}^{\prime}$ of flat $S$-linear categories induces an equivalence

$$
\delta: \operatorname{def}_{\mathfrak{b}} \longrightarrow \operatorname{def}_{\mathfrak{b}^{\prime}} ;
$$

(3) for every flat abelian $\mathcal{D}$ there is an equivalence

$$
\theta: \operatorname{Def}_{\mathcal{D}} \longrightarrow \operatorname{Def}_{\mathcal{D}^{\text {op }}} \text {; }
$$

(4) an equivalence $F: \mathcal{D} \longrightarrow \mathcal{D}^{\prime}$ of flat abelian categories induces an equivalence

$$
\delta: \operatorname{Def}_{\mathcal{D}^{\prime}} \longrightarrow \operatorname{Def}_{\mathcal{D}^{\prime}}
$$

Proof. The only non-trivial point here is that by Proposition 3.3, the opposite of a flat abelian category is again flat. Note that the corresponding statement for $R$-linear categories is obviously true.

8.5. Ind-objects versus coherent objects. If $\mathcal{C}_{e s}$ is essentially small, then there is an equivalence $\mathcal{C}_{e s} \cong \mathrm{Fp}\left(\operatorname{Ind}\left(\mathcal{C}_{e s}\right)\right)$. Similarly for a locally coherent Grothendieck category $\mathcal{C}$ there is an equivalence $\operatorname{lnd}(\operatorname{Fp}(\mathcal{C})) \cong \mathcal{C}$ (see 92.2$)$.

Using Propositions 5.5 and 5.6, we obtain pseudonatural transformations

$$
\operatorname{Def}_{\mathcal{D}_{e s}} \longrightarrow \operatorname{Def}_{\operatorname{lnd}\left(\mathcal{D}_{e s}\right)}
$$

and

$$
\operatorname{Def}_{\mathcal{D}} \longrightarrow \operatorname{Def}_{\mathrm{Fp}(\mathcal{D})}
$$

for an essentially small $S$-linear category $\mathcal{D}_{e s}$ and an $S$-linear locally coherent Grothendieck category $\mathcal{D}$. By Proposition 6.9, any $\mathcal{C} \in \operatorname{Def}_{\mathcal{D}_{e s}}$ is essentially small, and by Theorem 6.36, any $\mathcal{C} \in \operatorname{Def}_{\mathcal{D}}$ is a locally coherent Grothendieck category. This allows one to prove the following.

Theorem 8.8. (8.1) and (8.2) are equivalences of deformation pseudofunctors.

8.6. Lifting deformations of localizations. In this section we prove a general result (Theorem 8.14 below) which will allow us to construct deformation equivalences in several settings later in this paper. Unfortunately the formulation of Theorem 8.14 is rather technical, so the interested reader may consider reading the subsequent sections first.

Our purpose is to describe a class of functors $v: \mathfrak{v} \longrightarrow \mathcal{D}$, with $\mathfrak{v}$ flat pre-additive and $\mathcal{D}$ flat abelian, for which $\operatorname{def}_{\mathfrak{v}}$ and $\operatorname{Def}_{\mathcal{D}}$ are equivalent.

Consider a diagram

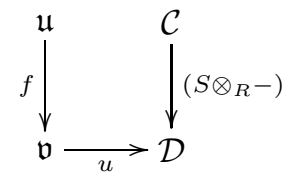


in which $v$ is an $S$-linear functor from a small $S$-linear category to an $S$-linear Grothendieck category, $\left(S \otimes_{R}-\right)$ is left adjoint to an abelian $R$-deformation $F$ : $\mathcal{D} \longrightarrow \mathcal{C}$ and $f$ is a linear $R$-deformation. In $\$ 7.1$ we have concentrated on lifting properties of $v$, a lift of $v$ being given. Now we will concentrate on the problem of lifting $v$. We have the following interpretation.

Proposition 8.9. The canonical functor

$$
\operatorname{Add}(S)(\mathfrak{v}, \mathcal{D}) \underset{\cong}{\longrightarrow} \operatorname{Add}(R)(\mathfrak{u}, \mathcal{D}) \underset{(F \circ \cdot)}{\longrightarrow} \operatorname{Add}(R)(\mathfrak{u}, \mathcal{C})
$$

is an abelian $R$-deformation, its left adjoint being given by

$$
\operatorname{Add}(R)(\mathfrak{u}, \mathcal{C}) \underset{\left.\left(S \otimes_{R}\right) \cdot\right)}{\longrightarrow} \operatorname{Add}(R)(\mathfrak{u}, \mathcal{D}) \underset{ }{\longrightarrow} \operatorname{Add}(S)(\mathfrak{v}, \mathcal{D})
$$

Hence an $R$-linear $u: \mathfrak{u} \longrightarrow \mathcal{C}$ making the diagram commute is a lift of $v$ along this left adjoint.

Proposition 8.10. Consider an $S$-linear functor $v: \mathfrak{v} \longrightarrow \mathcal{D}$ from a small $S$-linear category to an $S$-linear Grothendieck category such that the objects $v(V)$ are flat. Suppose $\mathrm{Ob}(\mathfrak{v})$ is endowed with a reflexive, transitive relation $\mathcal{R}$ such that

(1) $(U, V)$ not in $\mathcal{R}$ implies $\mathfrak{v}(U, V)=0$;

(2) $(U, V)$ in $\mathcal{R}$ implies that $v_{(U, V)}: \mathfrak{v}(U, V) \longrightarrow \mathcal{D}(v(U), v(V))$ is an isomorphism and that $\operatorname{Ext}_{\mathcal{D}}^{i}\left(v(U), M \otimes_{S} v(V)\right)=0$ for $M \in \bmod (S)$ and $i=1,2$. Consider a flat nilpotent $R$-deformation $F: \mathcal{D} \longrightarrow \mathcal{C}$. The following hold:

(1) There is a strict flat left $R$-deformation $f: \mathfrak{u} \longrightarrow \mathfrak{v}$ and an $R$-linear functor $u: \mathfrak{u} \longrightarrow \mathcal{C}$ for which all objects $u(U)$ are flat, such that the diagram

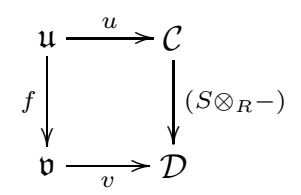

commutes up to natural isomorphism.

(2) If $f^{\prime}: \mathfrak{u}^{\prime} \longrightarrow \mathfrak{v}$ and $u^{\prime}: \mathfrak{u}^{\prime} \longrightarrow \mathcal{C}$ are like $f$ and $u$ (but with $f^{\prime}$ not necessarily strict), there is an equivalence $w: \mathfrak{u}^{\prime} \longrightarrow \mathfrak{u}$ with $f \circ w=f^{\prime}$ and $v \circ w \cong v^{\prime}$.

Proof. We will construct $u$ as stated in case $I^{2}=0$. It will be clear from the proof and from Proposition 6.13 that the $R$-linear functor $u: \mathfrak{u} \longrightarrow \mathcal{C}$ satisfies the same properties as $v$, which then finishes the proof in the general case.

For (1), consider a diagram

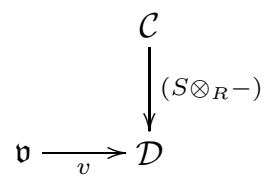

as stated. By Theorem 6.12, $v(V)$ has a flat lift $D_{V} \longrightarrow v(V)$ along the functor $\left(S \otimes_{R}-\right)$. Define $\mathfrak{u}$ by $\mathrm{Ob}(\mathfrak{u})=\mathrm{Ob}(\mathfrak{v})$ and $\mathfrak{u}(U, V)=\mathcal{C}\left(D_{U}, D_{V}\right)$ if $(U, V)$ is in $\mathcal{R}$ and $\mathfrak{u}(U, V)=0$ otherwise. Since $\mathcal{R}$ is transitive, there is an obvious composition on $\mathfrak{u}$ making $\mathfrak{u}$ into an $R$-linear category with an $R$-linear functor $u: \mathfrak{u} \longrightarrow \mathcal{C}$. There is also an obvious functor $f: \mathfrak{u} \longrightarrow \mathfrak{v}$. For $(U, V)$ not in $\mathcal{R}, f_{(U, V)}: 0=$ $\mathfrak{u}(U, V) \longrightarrow \mathfrak{v}(U, V)=0$ is trivially a deformation of modules. For $(U, V)$ in $\mathcal{R}$, 
$f_{(U, V)}$ is isomorphic to $\left(S \otimes_{R}-\right)_{(U, V)}: \mathcal{C}\left(D_{U}, D_{V}\right) \longrightarrow \mathcal{D}\left(S \otimes_{R} D_{U}, S \otimes_{R} D_{V}\right)$. It follows from Proposition 2.9 (8) that $\mathfrak{v}(U, V)$ is flat and $f_{(U, V)}$ is a deformation of modules. Hence $f$ is a flat left $R$-deformation, and the diagram commutes up to natural isomorphism.

For (2), consider $f^{\prime}$ and $u^{\prime}$ as stated. Since every $u^{\prime}(W)$ is flat and we have natural isomorphisms $S \otimes_{R} u^{\prime}(W) \longrightarrow v\left(f^{\prime}(W)\right)$, Theorem6.12 provides $\mathcal{D}$-isomorphisms $u^{\prime}(W) \longrightarrow D_{f^{\prime}(W)}$ that allow the definition of a functor $w: \mathfrak{u}^{\prime} \longrightarrow \mathfrak{u} . w$ is essentially surjective since $f^{\prime}$ is a deformation. For $\left(f^{\prime}(U), f^{\prime}(V)\right)$ not in $\mathcal{R}$, since $f_{(U, V)}^{\prime}: \mathfrak{u}^{\prime}(U, V) \longrightarrow \mathfrak{v}\left(f^{\prime}(U), f^{\prime}(V)\right)=0$ is a nilpotent deformation of modules, it follows that $\mathfrak{u}^{\prime}(U, V)=0$. Next, suppose $\left(f^{\prime}(U), f^{\prime}(V)\right)$ is in $\mathcal{R}$. We have a commutative diagram

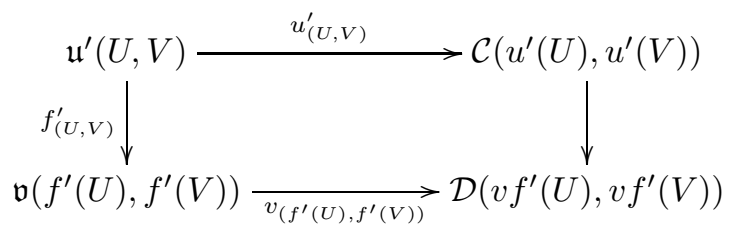

in which the right arow is isomorphic to $\left(S \otimes_{R}-\right)_{\left(u^{\prime}(U), u^{\prime}(V)\right)}$ and hence both vertical arrows are flat deformations of modules and $v_{\left(f^{\prime}(U), f^{\prime}(V)\right)}$ is an isomorphism. Since $I \otimes_{R} u_{(U, V)}^{\prime} \cong I \otimes_{S} v_{\left(f^{\prime}(U), f^{\prime}(V)\right)}$, it follows from the 5-lemma that $u_{(U, V)}^{\prime}$ and hence also $w_{(U, V)}$ is an isomorphism. This proves that $w$ is fully faithful.

Proposition 8.11. For $v: \mathfrak{v} \longrightarrow \mathcal{D}$ as in Proposition 8.10 and $R \in \mathrm{Rng}^{0} / S$, there is a functor

$$
\kappa_{R}: \operatorname{Def}_{\mathcal{D}}(R) \longrightarrow \operatorname{def}_{\mathfrak{v}}(R) .
$$

Proof. For $F: \mathcal{D} \longrightarrow \mathcal{C}$ in $\operatorname{Def}_{\mathcal{D}}(R)$, we can define $\kappa_{R}(F)$ as $f: \mathfrak{u} \longrightarrow \mathfrak{v}$ of Proposition 8.10(1). Proposition 8.10(2) can be used to define $\kappa_{R}$ on morphisms.

Proposition 8.12. For a localization of S-linear abelian categories

$$
\mathcal{D} \underset{i}{\stackrel{a}{\rightleftarrows}} \mathcal{K}
$$

and $R \in \mathrm{Rng}^{0} / S$, there is a functor

$$
\lambda_{R}: \operatorname{Def}_{\mathcal{D}}(R) \longrightarrow \operatorname{Def}_{\mathcal{K}}(R) .
$$

Proof. Since every localization factors as an equivalence followed by a strict localization, this is a consequence of Theorem 7.1 and Lemma 8.13 below applied to the functor $a$.

Lemma 8.13. Consider an $R$-linear exact functor $F: \mathcal{C} \longrightarrow \mathcal{D}$ that is essentially surjective. If $\mathcal{C}$ is flat over $R$, the same holds for $\mathcal{D}$.

Proof. For $D \in \mathcal{D}$ and $X \in \bmod (R)$, take an isomorphism $d: D \longrightarrow F(C)$ for a $C$ in $\mathcal{C}$. Take a $\mathcal{C}$-monomorphism $c: C \longrightarrow C^{\prime}$ with $\operatorname{Ext}_{\mathcal{C}, R}^{1}(X, c)=0$. Then $F(c) \circ d$ is a $\mathcal{D}$-monomorphism and by Proposition 2.7 $\operatorname{Ext}_{\mathcal{D}, R}^{1}(X, F(c))=F\left(\operatorname{Ext}_{\mathcal{C}, R}^{1}(X, c)\right)=$ 0 . 
The construction of an abelian deformation of $\operatorname{Mod}(\mathfrak{b})$ from an $R$-linear deformation of an essentially small category $\mathfrak{b}$ yields a pseudonatural transformation

$$
\mu: \operatorname{def}_{\mathfrak{b}} \longrightarrow \operatorname{Def}_{\operatorname{Mod}(\mathfrak{b})} \text {. }
$$

Theorem 8.14. Let $v: \mathfrak{v} \longrightarrow \mathcal{D}$ be as in Proposition 8.10 and suppose $v$ induces a localization

$$
\operatorname{Pr}(\mathfrak{v}) \stackrel{a}{\underset{i}{\rightleftarrows}} \mathcal{C} \text {. }
$$

The functors

$$
\operatorname{def}_{\mathfrak{v}}(R) \underset{\theta_{R}}{\longrightarrow} \operatorname{def}_{\mathfrak{v} \text { op }}(R) \underset{\mu_{R}}{\longrightarrow} \operatorname{Def}_{\operatorname{Pr}(\mathfrak{v})}(R) \underset{\lambda_{R}}{\longrightarrow} \operatorname{Def}_{\mathcal{D}}(R)
$$

and

$$
\operatorname{Def}_{\mathcal{D}}(R) \underset{\kappa_{R}}{\longrightarrow} \operatorname{def}_{\mathfrak{v}}(R)
$$

are inverse equivalences, where $\lambda_{R}$ is as in Proposition 8.12 , $\kappa_{R}$ as in Proposition 8.11. $\theta_{R}$ as in Proposition 8.7 and $\mu_{R}$ as above.

Proof. First, we indicate a natural isomorphism $\lambda_{R} \circ \mu_{R} \circ \theta_{R} \circ \kappa_{R} \cong 1_{\operatorname{Def}_{\mathcal{D}}(R)}$. For $F: \mathcal{D} \longrightarrow \mathcal{C}$ in $\operatorname{Def}_{\mathcal{D}}(R)$, its image $F^{\prime}$ under $\mu_{R} \circ \theta_{R} \circ \kappa_{R}$ fits into a diagram

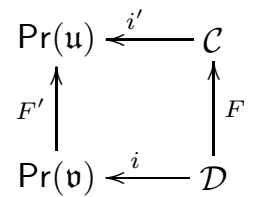

that commutes up to natural isomorphism. By Proposition 7.4 , $i^{\prime}$ is a localization. The construction of $\lambda_{R}\left(F^{\prime}\right)$ yields a diagram

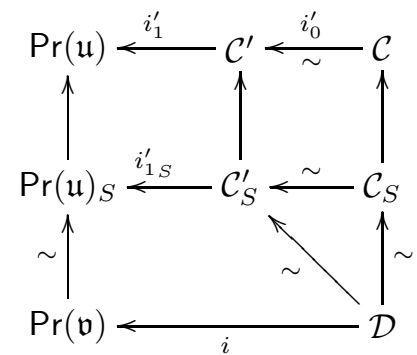

in which $i_{1}^{\prime}$ and $i_{1 S}^{\prime}$ are strict localizations and $i_{0}^{\prime}$ constitutes an equivalence of deformations between $F$ and $\lambda_{R}\left(F^{\prime}\right)$.

Next, we indicate a natural isomorphism $\kappa_{R} \circ \lambda_{R} \circ \mu_{R} \circ \theta_{R} \cong 1_{\text {def }_{\mathfrak{v}}(R)}$. Consider $f^{\prime}: \mathfrak{u}^{\prime} \longrightarrow \mathfrak{v}$ in $\operatorname{def}_{\mathfrak{v}}(R)$. Its image $F: \mathcal{D} \longrightarrow \mathcal{C}$ under $\lambda_{R} \circ \mu_{R} \circ \theta_{R}$ corresponds to a localization $i^{\prime}: \mathcal{C} \longrightarrow \operatorname{Pr}\left(\mathfrak{u}^{\prime}\right)$. The diagram

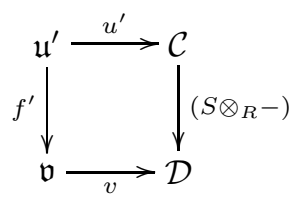

in which $u^{\prime}$ is induced by a left adjoint of $i^{\prime}$ and $\left(S \otimes_{R}-\right)$ is a left adjoint of $F$ commutes up to natural isomorphism. The functor $w: \mathfrak{u}^{\prime} \longrightarrow \mathfrak{u}$ of Proposition 8.10 constitutes an equivalence of deformations between $f^{\prime}$ and $\kappa_{R}(F)$. 
8.7. Deformations of Grothendieck categories with acyclic generators. Next we will formulate some specializations of Theorem 8.14

Theorem 8.15. Consider a flat $S$-linear Grothendieck category $\mathcal{D}$ and a small full subcategory $\mathfrak{g}$ such that $\mathrm{Ob}(\mathfrak{g})$ is a collection of flat generators of $\mathcal{D}$ and such that for $G, G^{\prime}$ in $\mathfrak{g}$

$$
\operatorname{Ext}_{\mathcal{D}}^{i}\left(G, M \otimes_{S} G^{\prime}\right)=0
$$

for $i=1,2$ and $M \in \bmod (S)$. For $R \in \operatorname{Rng}^{0} / S$, there is an equivalence

$$
\operatorname{def}_{\mathfrak{g}}(R) \cong \operatorname{Def}_{\mathcal{D}}(R) \text {. }
$$

Proof. It is readily seen that the inclusion $\mathfrak{g} \longrightarrow \mathcal{D}$ satisfies the conditions of Theorem 8.14 .

Theorem 8.16. For an essentially small flat $S$-linear category $\mathfrak{b}$,

$$
\mu: \operatorname{def}_{\mathfrak{b}} \longrightarrow \operatorname{Def}_{\operatorname{Mod}(\mathfrak{b})}
$$

is an equivalence of deformation pseudofunctors.

Proof. Since $\mathfrak{b}^{\text {op }}$ is a full subcategory of $\operatorname{Mod}(\mathfrak{b})$ satisfying the conditions of Theorem 8.15, the result follows from Theorem 8.14 .

8.8. Deformations of categories with enough injectives. We have seen in Proposition 6.25 that if $\mathcal{C}$ is an abelian category with enough injectives, there is an equivalence $\mathcal{C} \cong(\bmod (\operatorname{Inj}(\mathcal{C})))^{\mathrm{op}}$. We prove the following.

Theorem 8.17. If $\mathcal{D}$ has enough injectives, then $\operatorname{Def}_{\mathcal{D}}$ and $\operatorname{def}_{\operatorname{lnj}(\mathcal{D})}$ are equivalent deformation pseudofunctors.

Proof. Since $\bmod (\operatorname{Inj}(\mathcal{C})) \cong \mathcal{V}-\operatorname{Fp}(\mathcal{V}-\operatorname{Mod}(\operatorname{Inj}(\mathcal{C})))$ where $\operatorname{Inj}(\mathcal{C}) \in \mathcal{V}$, we obtain a pseudonatural transformation

$$
\operatorname{def}_{\operatorname{lnj}(\mathcal{C})} \longrightarrow \operatorname{Def}_{(\bmod (\operatorname{Inj}(\mathcal{C})))^{\text {op }},}
$$

which is actually an equivalence by Theorems 8.16, 8.8 and Proposition 8.7.

In Appendix A, we clarify this equivalence a little further using certain preservation properties of flat nilpotent linear deformations.

8.9. Sheaves of modules over a ringed space. We will now give an application of Theorem 8.14 to sheaves of modules over a ringed space.

Let $X$ be a topological space and let $\mathcal{O}_{X}$ be a sheaf of $S$-algebras on $X$. For an open $U \subset X$, let $\mathcal{O}_{U}$ be the restriction of $\mathcal{O}_{X}$ to $U$ and denote by $\operatorname{Mod}\left(\mathcal{O}_{U}\right)$ and $\operatorname{PreMod}\left(\mathcal{O}_{U}\right)$ the $(S$-linear $)$ categories of sheaves and presheaves of $\mathcal{O}_{U}$-modules. For a basis $\mathcal{B}$ of the topology, denote by $\mathcal{O}_{\mathcal{B}}$ the restriction of $\mathcal{O}_{X}$ to $\mathcal{B}$ and by $\operatorname{PreMod}\left(\mathcal{O}_{\mathcal{B}}\right)$ the category of presheaves of $\mathcal{O}_{\mathcal{B}}$-modules.

Theorem 8.18. Let $X$ be a topological space and let $\mathcal{O}_{X}$ be a sheaf of flat $S$ algebras on $X$ as above. Suppose the topology has a basis $\mathcal{B}$ such that for every $U \in \mathcal{B}$, the sheaf cohomology

$$
H^{i}\left(U, M \otimes_{S} \mathcal{O}_{U}\right)=0
$$

for $i=1,2$ and $M \in \bmod (S)$. For $R \in \operatorname{Rng}^{0} / S$, there is an equivalence

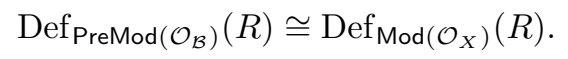


Proof. Let $a: \operatorname{PreMod}\left(\mathcal{O}_{X}\right) \longrightarrow \operatorname{Mod}\left(\mathcal{O}_{X}\right)$ be the exact sheafication functor left adjoint to inclusion. For an open $U \subset X$, let $j_{U}: U \longrightarrow X$ be the inclusion map and let $P_{U}=j_{U, !}^{p} \mathcal{O}_{U}$ and $S_{U}=j_{U, !} \mathcal{O}_{U}=a\left(j_{U, !}^{p} \mathcal{O}_{U}\right)$ be the extensions by zero of $\mathcal{O}_{U}$ in the categories of presheaves and sheaves. Also, let $P_{U}^{b}$ denote the restriction of $P_{U}$ to $\mathcal{B}$. For any basis $\mathcal{B}$, for $U \in \mathcal{B}$ and $F \in \operatorname{PreMod}\left(\mathcal{O}_{\mathcal{B}}\right)$, we have $\operatorname{Hom}\left(P_{U}^{b}, F\right)=F(U)$. For $U$ and $V$ in $\mathcal{B}$, we obtain

$$
\operatorname{Hom}\left(P_{U}^{b}, P_{V}^{b}\right)=\operatorname{Hom}\left(P_{U}, P_{V}\right)=\left\{\begin{aligned}
\mathcal{O}_{X}(U) & \text { if } U \subset V \\
0 & \text { otherwise }
\end{aligned}\right.
$$

and

$$
\operatorname{Hom}\left(S_{U}, S_{V}\right)=\left\{f \in \mathcal{O}_{X}(U) \mid f \text { is zero on a neighbourhood of } U \backslash V\right\} .
$$

Thus in particular if $U \subset V$,

$$
\operatorname{Hom}\left(P_{U}, P_{V}\right)=\operatorname{Hom}\left(S_{U}, S_{V}\right) .
$$

Let $\mathfrak{u}$ be the full subcategory of $\operatorname{PreMod}\left(\mathcal{O}_{X}\right)$ spanned by the objects $P_{U}$ for $U \in$ $\mathcal{B}$. By (8.4), $\mathfrak{u}$ is isomorphic to the full subcategory of $\operatorname{PreMod}\left(\mathcal{O}_{\mathcal{B}}\right)$ spanned by the objects $P_{U}^{b}$ for $U \in \mathcal{B}$. Since these objects are a family of finitely generated projective generators of $\operatorname{PreMod}\left(\mathcal{O}_{\mathcal{B}}\right)$, we deduce that

$$
\operatorname{PreMod}\left(\mathcal{O}_{\mathcal{B}}\right) \cong \operatorname{Pr}(\mathfrak{u})
$$

hence it suffices to prove that

$$
\operatorname{def}_{\mathfrak{u}}(R) \cong \operatorname{Def}_{\operatorname{Mod}\left(\mathcal{O}_{X}\right)}(R) .
$$

Let

$$
u: \mathfrak{u} \longrightarrow \operatorname{Mod}\left(\mathcal{O}_{X}\right)
$$

be the restriction of $a$. We will show that $u$ satisfies the conditions of Theorem 8.14, It is easily seen that $u$ induces a localization (see for example [15]), so it remains to verify the conditions stated in Proposition [8.10. If we say that $\left(P_{U}, P_{V}\right)$ is in $\mathcal{R}$ if $U \subset V$, the result follows from (8.4), (8.6) and the following computation for $U \subset V:$

$$
\begin{aligned}
\operatorname{Ext}_{\operatorname{Mod}\left(\mathcal{O}_{X}\right)}^{i}\left(S_{U}, M \otimes_{S} S_{V}\right) & =\operatorname{Ext}_{\operatorname{Mod}\left(\mathcal{O}_{U}\right)}^{i}\left(\mathcal{O}_{U},\left.\left(M \otimes_{S} S_{V}\right)\right|_{U}\right) \\
& =\operatorname{Ext}_{\operatorname{Mod}\left(\mathcal{O}_{U}\right)}^{i}\left(\mathcal{O}_{U}, M \otimes_{S} \mathcal{O}_{U}\right) \\
& =H^{i}\left(U, M \otimes_{S} \mathcal{O}_{U}\right),
\end{aligned}
$$

where we have used that $j_{U, !}$ is exact and hence its right adjoint restriction functor $(-)_{U}$ preserves injectives.

\section{Appendix A.}

In this appendix we indicate a direct proof of Theorem 8.17. This proof makes use of some preservation properties of flat nilpotent linear deformations that may be of independent interest. 
A.1. Flat nilpotent linear deformations. Let $F: \mathfrak{a} \longrightarrow \mathfrak{b}$ be a flat nilpotent $R$-deformation of an $S$-linear category $\mathfrak{b}$. In this section, we will lift some properties of $\mathfrak{b}$ to $\mathfrak{a}$. The following is well known in the ring case.

Proposition A.1. If $f: A_{1} \longrightarrow A_{2}$ in $\mathfrak{a}$ is such that $F(f)$ is an isomorphism in $\mathfrak{b}$, then $f$ is an isomorphism in $\mathfrak{a}$.

Proof. It suffices to consider $\mathfrak{a} \longrightarrow S \otimes_{R} \mathfrak{a}$ nilpotent of order 2. Suppose $f: A_{1} \longrightarrow$ $A_{2}$ in $\mathfrak{a}$ is an isomorphism in $\mathfrak{b}$. Take an $\mathfrak{a}$-map $g: A_{2} \longrightarrow A_{1}$ such that $g \circ f=1_{A_{1}}$ and $f \circ g=1_{A_{2}}$ in $S \otimes_{R} \mathfrak{a}$. From the exact rows $0 \longrightarrow I \mathfrak{a}\left(A, A^{\prime}\right) \longrightarrow \mathfrak{a}\left(A, A^{\prime}\right) \longrightarrow$ $\left(S \otimes_{R} \mathfrak{a}\right)\left(A, A^{\prime}\right) \longrightarrow 0$ and from $I^{2}=0$ we deduce that

$$
\left(g \circ f-1_{A_{1}}\right)^{2}=0
$$

and

$$
\left(f \circ g-1_{A_{2}}\right)^{2}=0 .
$$

These equations can be rewritten as

$$
(2 g-g \circ f \circ g) \circ f=1_{A_{1}}
$$

and

$$
f \circ(2 g-g \circ f \circ g)=1_{A_{2}},
$$

which proves our assertion.

Proposition A.2. If $Z \in \mathfrak{a}$ is such that $F(Z)$ is a zero-object in $\mathfrak{b}$, then $Z$ is a zero-object in $\mathfrak{a}$.

Proof. It suffices to consider $\mathfrak{a} \longrightarrow S \otimes_{R} \mathfrak{a}$. Suppose $Z$ is a zero-object in $S \otimes_{R} \mathfrak{a}$. So $\left(S \otimes_{R} \mathfrak{a}\right)(A, Z)=0$ for all $\mathfrak{a}$-objects $A$. But then by Nakayama $\mathfrak{a}(A, Z)=0$ for all $A$, meaning that $Z$ is a zero-object in $\mathfrak{a}$.

Definition A.3. In a category $\mathcal{C}$, an idempotent is a map $e$ with $e \circ e=e$. An idempotent $e$ splits if there exist maps $r, s$ with $e=s \circ r$ and $r \circ s=1$. A category in which all idempotents split is called Karoubian.

Remark A.4. The splitting of an idempotent $e$ is equivalent to the existence of the equalizer of $e$ and 1 and to the existence of the coequalizer of $e$ and 1. Thus an abelian category is Karoubian. If $\mathcal{C}$ is an abelian category with enough injectives, $\operatorname{lnj}(\mathcal{C})$ is Karoubian too since a retract of an injective is injective.

Proposition A.5. If $\mathfrak{b}$ is Karoubian, the same holds for $\mathfrak{a}$.

Proof. It suffices to consider $\mathfrak{a} \longrightarrow S \otimes_{R} \mathfrak{a}$ nilpotent of order 2. Let $e: A \longrightarrow A$ be an idempotent in $\mathfrak{a}$ and take maps $s: B \longrightarrow A, r: A \longrightarrow B$ in $\mathfrak{a}$ such that $s \circ r=e$ and $r \circ s=1$ in $S \otimes_{R} \mathfrak{a}$. From $s \circ r-e \in I \mathfrak{a}(A, A)$ and $r \circ s-1 \in I \mathfrak{a}(B, B)$ we obtain the following equations in $\mathfrak{a}$ :

(1) $(s \circ r-e)^{2}=0$;

(2) $(r \circ s-1)^{2}=0$

(3) $(r \circ s-1) \circ r \circ(s \circ r-e)=0$;

(4) $(s \circ r-e) \circ s \circ(r \circ s-1)=0$.

It follows from (1) that

$$
(s+e \circ s-s \circ r \circ s) \circ(r \circ e)=e
$$

and from (2) that

$$
2(r \circ s)-r \circ s \circ r \circ s=1 .
$$


Combining (1) and (3) gives us

$$
r \circ e \circ s \circ r=r \circ s \circ r
$$

and combining this with (2) and (4) gives

$$
r \circ e \circ s=r \circ s
$$

We can now compute that $(r \circ e) \circ(s+e \circ s-s \circ r \circ s)=2(r \circ s)-r \circ s \circ r \circ s=1$. So we have shown that $(s+e \circ s-s \circ r \circ s): B \longrightarrow A$ and $(r \circ e): A \longrightarrow B$ give a splitting of $e$ in $\mathfrak{a}$.

Proposition A.6. If $A, B, C, s_{1}: A \longrightarrow C$ and $s_{2}: B \longrightarrow C$ in $\mathfrak{a}$ are such that $\left(F(C), F\left(s_{1}\right), F\left(s_{2}\right)\right)$ is a coproduct of $F(A)$ and $F(B)$ in $\mathfrak{b}$, then $\left(C, s_{1}, s_{2}\right)$ is a coproduct of $A$ and $B$ in $\mathfrak{a}$.

Proof. It suffices to consider $\mathfrak{a} \longrightarrow S \otimes_{R} \mathfrak{a}$ nilpotent of order 2. Take maps $p_{1}$ : $C \longrightarrow A$ and $p_{2}: C \longrightarrow B$ in $\mathfrak{a}$ such that $\left(C, s_{1}, s_{2}, p_{1}, p_{2}\right)$ is a biproduct of $A$ and $B$ in $S \otimes_{R} \mathfrak{a}$. We obtain the following equations in $\mathfrak{a}$ :

(1) $\left(p_{1} \circ s_{1}-1\right)^{2}=0$

(2) $\left(p_{1} \circ s_{2}\right) \circ\left(p_{2} \circ s_{1}\right)=0$;

(3) $\left(p_{1} \circ s_{1}-1\right) \circ\left(p_{1} \circ s_{2}\right)=0$;

(4) $\left(p_{1} \circ s_{2}\right) \circ\left(p_{2} \circ s_{2}-1\right)=0$;

(5) $\left(s_{1} \circ p_{1}+s_{2} \circ p_{2}-1\right)^{2}=0$.

Put

and

$$
p_{1}^{\prime}=2 p_{1}-p_{1} \circ s_{1} \circ p_{1}-p_{1} \circ s_{2} \circ p_{2}
$$

$$
p_{2}^{\prime}=2 p_{2}-p_{2} \circ s_{2} \circ p_{2}-p_{2} \circ s_{1} \circ p_{1} .
$$

It follows from (1) and (2) that $p_{1}^{\prime} \circ s_{1}=2 p_{1} \circ s_{1}-\left(2 p_{1} \circ s_{1}-1\right)-0=1$. From (3) and (4) we find that $p_{1}^{\prime} \circ s_{2}=2 p_{1} \circ s_{2}-p_{1} \circ s_{2}-p_{1} \circ s_{2}=0$. Finally, using (5) we obtain that $s_{1} \circ p_{1}^{\prime}+s_{2} \circ p_{2}^{\prime}=1$. Combining these results with their symmetric results (changing the roles of $A$ and $B$ ), we have shown that $\left(C, s_{1}, s_{2}, p_{1}^{\prime}, p_{2}^{\prime}\right)$ is a biproduct of $A$ and $B$ in $\mathfrak{a}$, which proves our assertion.

Proposition A.7. If $\mathfrak{b}$ is additive, the same holds for $\mathfrak{a}$.

Proof. This immediately follows from Proposition A.2 and Proposition A.6.

Definition A.8. A pre-additive category $\mathfrak{a}$ is called coherent if $\bmod (\mathfrak{a})$ is abelian.

Proposition A.9. If $\mathfrak{b}$ is coherent, the same holds for $\mathfrak{a}$.

Proof. We may assume that $\mathfrak{a}, \mathfrak{b}$ are small. Then the result follows from Proposition 5.4 and Theorem 6.36.

We mention the following intrinsic characterization of coherence, which we will not explicitly use.

Definition A.10 (14]). Consider a map $f: C \longrightarrow C^{\prime}$ in a pre-additive category $\mathcal{C}$. A weak cokernel of $f$ is a map $g: C^{\prime} \longrightarrow C^{\prime \prime}$ with $g \circ f=0$ and such that every map $h$ with $h \circ f=0$ factorizes as $h=h^{\prime} \circ g$.

In a triangulated category, the cone of a morphism is a weak cokernel.

Proposition A.11 ([14, Lemma 1]). An additive category $\mathfrak{a}$ is coherent if and only if $\mathfrak{a}$ has weak cokernels. 
Remark A.12. If $\mathcal{C}$ is an abelian category with enough injectives, there is an equivalence $\mathcal{C} \cong(\bmod (\operatorname{Inj}(\mathcal{C})))^{\text {op }}$ (see Proposition 6.25), hence $\operatorname{Inj}(\mathcal{C})$ is coherent. For a map between injectives, we can first take its cokernel and then a mono to an injective to obtain a weak cokernel in $\operatorname{lnj}(\mathcal{C})$.

A.2. Deformations with enough injectives. We have seen in Theorem 8.17 that if $\mathcal{C}$ is an abelian category with enough injectives, there is an equivalence $\operatorname{Def}_{\mathcal{C}} \cong \operatorname{def}_{\operatorname{Inj}(\mathcal{C})}$. We will now give a different approach to this fact. We start by characterizing "categories of injectives of abelian categories with enough injectives".

Proposition A.13. For an abelian category $\mathcal{C}$ with enough injectives, $\operatorname{lnj}(\mathcal{C})$ is an additive, coherent, Karoubian category.

Proof. $\operatorname{Inj}(\mathcal{C})$ is additive since products of injectives are injective, and we already noted in Remark A.4 that $\operatorname{Inj}(\mathcal{C})$ is Karoubian and in $\operatorname{Remark}$ A.12 that $\operatorname{Inj}(\mathcal{C})$ is coherent.

Proposition A.14. For an additive, coherent, Karoubian category $\mathfrak{a}$, the functor

$$
\mathfrak{a}^{\mathrm{op}} \longrightarrow \bmod (\mathfrak{a}): A \longmapsto(A,-)
$$

induces an equivalence of categories

$$
\mathfrak{a}^{\text {op }} \longrightarrow \mathfrak{p}
$$

to the full subcategory $\mathfrak{p}$ of projectives of $\bmod (\mathfrak{a})$.

Proof. Take an object $P$ of $\mathfrak{p}$ and consider a presentation

$$
\bigoplus_{i=1}^{n} \mathfrak{a}\left(A_{i},-\right) \longrightarrow \bigoplus_{j=1}^{m} \mathfrak{a}\left(A_{j},-\right) \longrightarrow P \longrightarrow 0
$$

Since $P$ is projective and $\mathfrak{a}$ is additive, $P$ is a retract of the functor $\mathfrak{a}\left(\bigoplus_{j=1}^{m} A_{j},-\right)$. But since $\mathfrak{a}$ is Karoubian, it follows that $P$ is itself representable.

In other words, for an additive, coherent, Karoubian category $\mathfrak{a}$, there is an equivalence $\mathfrak{a} \cong \operatorname{lnj}\left((\bmod (\mathfrak{a}))^{\mathrm{op}}\right)$. Combining this with Proposition A.13, we have thus characterized the "categories of injectives of abelian categories with enough injectives" as being precisely the additive, coherent, Karoubian categories.

Let $\mathfrak{b}$ be a coherent $S$-linear category. Since $\bmod (\mathfrak{b}) \cong \mathcal{V}-F p(\mathcal{V}-\operatorname{Mod}(\mathfrak{b}))$, where $\mathfrak{b} \in \mathcal{V}$, we obtain a pseudonatural transformation

$$
\operatorname{def}_{\mathfrak{b}} \longrightarrow \operatorname{Def}_{(\bmod (\mathfrak{b}))^{\mathrm{op}}}
$$

which is actually an equivalence by Theorem 8.16 . Theorem 8.8 and Proposition 8.7. Taking $\mathfrak{b}=\operatorname{Inj}(\mathcal{D})$, this is precisely the equivalence (8.3) of 88.8 . We will now suggest another proof of this equivalence.

Proposition A.15. Every flat, nilpotent abelian deformation $\mathcal{D} \longrightarrow \mathcal{C}$ with enough injectives induces a flat linear deformation $\operatorname{Hom}_{R}(S,-): \operatorname{lnj}(\mathcal{C}) \longrightarrow \operatorname{Inj}(\mathcal{D})$.

Proof. Since taking injectives preserves equivalence of categories, we may consider $\mathcal{C}_{S} \longrightarrow \mathcal{C}$. By Proposition 2.9(5) and Proposition 4.3, the map

$$
\mathcal{C}(C, E) \longrightarrow \mathcal{C}\left(\operatorname{Hom}_{R}(S, C), E\right) \longrightarrow \mathcal{C}\left(\operatorname{Hom}_{R}(S, C), \operatorname{Hom}_{R}(S, E)\right)
$$

induces an isomorphism $S \otimes_{R} \mathcal{C}(C, E) \longrightarrow \mathcal{C}\left(\operatorname{Hom}_{R}(S, C), \operatorname{Hom}_{R}(S, E)\right)$ when $E$ is injective. It then follows by Corollary 6.15 that $\operatorname{Hom}_{R}(S,-): \operatorname{Inj}(\mathcal{C})^{S} \longrightarrow \operatorname{Inj}\left(\mathcal{C}_{S}\right)$ is an equivalence. The flatness of $\operatorname{lnj}(\mathcal{C})$ follows from Proposition 2.9 (6) $)$. 
Let $\mathcal{D}$ be an abelian $S$-linear category with enough injectives. Proposition A.15 yields a pseudonatural transformation

$$
\operatorname{Def}_{\mathcal{D}} \longrightarrow \operatorname{def}_{\operatorname{lnj}(\mathcal{D})}
$$

By Theorem 6.16 any $\mathcal{C} \in \operatorname{Def}_{\mathcal{D}}$ has enough injectives, and by Propositions A.13, A.7 A.5 and A.9, any $\mathfrak{a} \in \operatorname{def}_{\mathfrak{b}}$ is an additive, coherent, Karoubian category. This allows one to prove the following.

Theorem A.16. (A.1) and (A.2) are equivalences of deformation pseudofunctors.

\section{Appendix B.}

In this appendix, we consider an alternative deformation pseudofunctor $\operatorname{def}_{\mathfrak{b}}^{s}$ that can be used to study linear deformations of an $S$-linear category $\mathfrak{b}$, and we study its relation with $\operatorname{def}_{\mathfrak{b}}$.

For an $S$-linear category $\mathfrak{b}$ and for $R \in \mathrm{Rng}^{0} / S$, consider the following groupoid $\operatorname{def}_{\mathfrak{b}}^{s}$ : the objects of $\operatorname{def}_{\mathfrak{b}}^{s}$ are strict $R$-deformations $\mathfrak{a} \longrightarrow \mathfrak{b}$. The morphisms of $\operatorname{def}_{\mathfrak{b}}^{s}$ are isomorphisms of deformations.

There are obvious functors

$$
\sigma_{R}: \operatorname{def}_{\mathfrak{b}}^{s}(R) \longrightarrow \operatorname{def}_{\mathfrak{b}}(R)
$$

constituting a pseudonatural transformation $\sigma: \operatorname{def}_{\mathfrak{b}}^{s} \longrightarrow \operatorname{def}_{\mathfrak{b}}$. As every functor between groupoids does, $\sigma_{R}$ induces a function

$$
\operatorname{Sk}\left(\sigma_{R}\right): \operatorname{Sk}\left(\operatorname{def}_{\mathfrak{b}}^{s}(R)\right) \longrightarrow \operatorname{Sk}\left(\operatorname{def}_{\mathfrak{b}}(R)\right) .
$$

We will show that for $R$ in $\operatorname{Rng}^{0} / S, \operatorname{Sk}\left(\sigma_{R}\right)$ is a bijection.

Proposition B.1. For $R$ in $\mathrm{Rng}^{0} / S, \sigma_{R}$ is full.

Proof. Consider two strict left $R$-deformations $f_{1}: \mathfrak{a}_{1} \longrightarrow \mathfrak{b}$ and $f_{2}: \mathfrak{a}_{2} \longrightarrow \mathfrak{b}$ and an equivalence of deformations $\varphi: \mathfrak{a}_{1} \longrightarrow \mathfrak{a}_{2}$. We will construct an isomorphism of deformations $\varphi^{\prime}: \mathfrak{a}_{1} \longrightarrow \mathfrak{a}_{2}$ that is naturally isomorphic to $\varphi$. Consider a natural isomorphism $\eta: f_{1} \longrightarrow f_{2} \circ \varphi$. For every a-map $a: A \longrightarrow A^{\prime}$, there is a commutative square

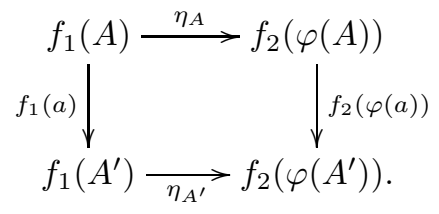

Since $f_{2}$ is a strict deformation, every $A$ in $\mathfrak{a}_{1}$ determines a unique object $\varphi^{\prime}(A)$ in $\mathfrak{a}_{2}$ satisfying

$$
f_{2}\left(\varphi^{\prime}(A)\right)=f_{1}(A)
$$

By Proposition A.1, we can lift the $\mathfrak{b}$-isomorphisms

$$
\eta_{A}: f_{2}\left(\varphi^{\prime}(A)\right) \longrightarrow f_{2}(\varphi(A))
$$

to $\mathfrak{a}_{2}$-isomorphisms

$$
\mu_{A}: \varphi^{\prime}(A) \longrightarrow \varphi(A)
$$


with $f_{2}\left(\mu_{A}\right)=\eta_{A}$. We can now define $\varphi^{\prime}(a)$ for $a: A \longrightarrow A^{\prime}$ in $\mathfrak{a}_{1}$ as the unique $\mathfrak{a}_{2}$-map making the following square commute:

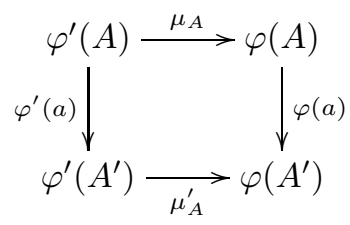

Clearly, $\varphi^{\prime}: \mathfrak{a}_{1} \longrightarrow \mathfrak{a}_{2}$ is a functor and $\mu: \varphi^{\prime} \longrightarrow \varphi$ is a natural isomorphism. If we apply $f_{2}$ to the second square and compare the resulting square with the first square, we conclude that $f_{2} \circ \varphi^{\prime}=f_{1}$, as we wanted.

Remark B.2. $\sigma_{R}$ need in general not be faithful. Indeed, consider any algebradeformation $f: A \longrightarrow B$ in which $A$ contains a non-central invertible element $\xi$ with $f(\xi)=1$. Then $\varphi: A \longrightarrow A: a \longmapsto \xi^{-1} a \xi$ is an algebra-isomorphism different from but naturally isomorphic to $1_{A}$ with $f \circ \varphi=f$.

Proposition B.3. For $R$ in $\mathrm{Rng}^{0} / S, \sigma_{R}$ is essentially surjective.

Proof. Consider a left $R$-deformation $f: \mathfrak{a} \longrightarrow \mathfrak{b}$. Take an inverse equivalence $g: \mathfrak{b} \longrightarrow S \otimes_{R} \mathfrak{a}$ of the canonical equivalence $S \otimes_{R} f: S \otimes_{R} \mathfrak{a} \longrightarrow \mathfrak{b}$. We construct a category $\mathfrak{c}$ in the following way: the objects of $\mathfrak{c}$ are precisely the objects of $\mathfrak{b}$. For objects $B, B^{\prime}$ of $\mathfrak{b}, \mathfrak{c}\left(B, B^{\prime}\right)$ is defined to equal $\mathfrak{a}\left(g(B), g\left(B^{\prime}\right)\right)$, and the composition in $\mathfrak{c}$ is the composition in $\mathfrak{a}$. There is an obvious functor $\varphi: \mathfrak{c} \longrightarrow \mathfrak{a}$ mapping $B$ to $g(B)$ and $a: g(B) \longrightarrow g\left(B^{\prime}\right)$ to $a: g(B) \longrightarrow g\left(B^{\prime}\right) . \varphi$ is clearly fully faithful. For $A$ in $\mathfrak{a}, \varphi(f(A))=g(f(A))$ is isomorphic to $A$ in $S \otimes_{R} \mathfrak{a}$. But by Proposition A.1. they remain isomorphic in $\mathfrak{a}$. It follows that $\varphi$ is essentially surjective and hence an equivalence of categories. Take a natural isomorphism $\eta: 1_{\mathfrak{b}} \longrightarrow S \otimes_{R} f \circ g$. We define a functor $h: \mathfrak{c} \longrightarrow \mathfrak{b}$ by putting $h(B)=B$ and mapping $a: g(B) \longrightarrow g\left(B^{\prime}\right)$ to the unique map $h(a)$ making the following diagram commute:

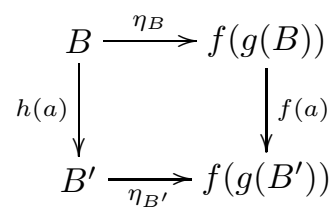

It follows that the $\eta$ define a natural isomorphism $h \longrightarrow f \circ \varphi$. Finally, we have to show that $S \otimes_{R} h: S \otimes_{R} \mathfrak{c} \longrightarrow \mathfrak{b}$ is an isomorphism. Since $S \otimes_{R} h$ is clearly bijective on objects, it suffices that $S \otimes_{R} h$ or equivalently $S \otimes_{R}(f \circ \varphi)$ is an equivalence, which is obvious.

Theorem B.4. For $R$ in $\operatorname{Rng}^{0} / S, \operatorname{Sk}\left(\sigma_{R}\right)$ is a bijection.

Proof. Sk $\left(\sigma_{R}\right)$ is injective by Proposition B.1 and surjective by Proposition B.3.

\section{REFERENCES}

[1] J. Adámek, H. Herrlich, and G. E. Strecker, Abstract and concrete categories, Pure and Applied Mathematics, John Wiley \& Sons, Inc., New York, 1990, The joy of cats, A WileyInterscience Publication. MR1051419 (91h:18001)

[2] M. Artin, A. Grothendieck, and J. L. Verdier, Theorie des topos et cohomologie étale des schémas, SGA4, Tome 3, Lecture Notes in Mathematics, vol. 305, Springer-Verlag, 1973. MR0354654(50:7132) 
[3] M. Artin and J. J. Zhang, Abstract Hilbert schemes, Algebr. Represent. Theory 4 (2001), no. 4, 305-394. MR:1863391 (2002h:16046)

[4] F. Borceux, Handbook of categorical algebra. 2, Encyclopedia of Mathematics and its Applications, vol. 51, Cambridge University Press, Cambridge, 1994, Categories and structures. MR1313497 (96g:18001b)

[5] N. Bourbaki, Eléments de mathématique. 22. Première partie: Les structures fondamentales de l'analyse. Livre 1: Théorie des ensembles. Chapitre 4: Structures, Actualités Sci. Ind. no. 1258, Hermann, Paris, 1957. MR0097335 (20:3804)

[6] M. Gerstenhaber and S. D. Schack, On the deformation of algebra morphisms and diagrams, Trans. Amer. Math. Soc. 279 (1983), no. 1, 1-50. MR0704600 (85d:16021)

[7] _ Algebraic cohomology and deformation theory, Deformation theory of algebras and structures and applications (Il Ciocco, 1986) (Dordrecht), NATO Adv. Sci. Inst. Ser. C Math. Phys. Sci., vol. 247, Kluwer Acad. Publ., Dordrecht, 1988, pp. 11-264. MR0981619 (90c:16016)

[8] The cohomology of presheaves of algebras. I. Presheaves over a partially ordered set, Trans. Amer. Math. Soc. 310 (1988), no. 1, 135-165. MR0965749 (89k:16052)

[9] M. Gerstenhaber, On the deformation of rings and algebras, Ann. of Math. (2) 79 (1964), 59-103. MR0171807(30:2034)

[10] - On the deformation of rings and algebras. II, Ann. of Math. 84 (1966), 1-19. MR0207793 (34:7608)

[11] A. Grothendieck, Sur quelques points d'algèbre homologiques, Tôhoku Math. J. (2) 9 (1957), 119-221. MR0102537 (21:1328)

[12] L. Illusie, Existence de résolutions globales, SGA6, Lecture Notes in Math., vol. 225, SpringerVerlag, 1971. MR0354655 (50:7133)

[13] H. Krause, The spectrum of a module category, Mem. Amer. Math. Soc. 149 (2001), no. 707, $\mathrm{x}+125$. MR 1803703 (2001k:16010)

[14] _ A Brown representability theorem via coherent functors, Topology 41 (2002), no. 4, 853-861. MR:1905842 (2003c:18011)

[15] W. Lowen, A generalization of the Gabriel-Popescu theorem, Journal Pure and Appl. Algebra 190 (2004), 197-211. MR2043328

[16] - Obstruction theory for objects in abelian and derived categories, Communications in Alg. 33 (2005), no. 9, 3195-3223. MR2175388

[17] W. Lowen and M. Van den Bergh, Hochschild cohomology of abelian categories and ringed spaces, Advances in Math. 198 (2005), 172-221. MR2183254

[18] S. MacLane, Categories for the working mathematician, Springer-Verlag, Berlin, 1971. MR0354798 (50:7275)

[19] B. Mitchell, Rings with several objects, Advances in Math. 8 (1972), 1-161. MR0294454 $(45: 3524)$

[20] A. Neeman, Triangulated categories, Annals of Mathematics Studies, vol. 148, Princeton University Press, Princeton, NJ, 2001. MR 1812507 (2001k:18010)

[21] N. Popesco and P. Gabriel, Caractérisation des catégories abéliennes avec générateurs et limites inductives exactes, C. R. Acad. Sci. Paris 258 (1964), 4188-4190. MR0166241 (29:3518)

[22] N. Popescu, Abelian categories with applications to rings and modules, Academic Press, London, 1973, London Mathematical Society Monographs, no. 3. MR0340375 (49:5130)

[23] L. A. Takhtadjian, Noncommutive homology of quantum tori, Functional Anal. Appl. 23 (1989), 147-149. MR1011367 (90m:18015)

Departement DWis, Vrije Universiteit Brussel, Pleinlaan 2, 1050 Brussel, Belgium E-mail address: wlowen@vub.ac.be

Departement Wni, Limburgs Universitair Centrum, Universitaire Campus, Building D, 3590 Diepenbeek, Belgium

E-mail address: vdbergh@luc.ac.be 